(c) Institute of Mathematical Statistics, 2003

\title{
THE VALUATION OF AMERICAN CALL OPTIONS ON THE MINIMUM OF TWO DIVIDEND-PAYING ASSETS ${ }^{1}$
}

\author{
By Jerome Detemple, Shui Feng And Weidong Tian \\ Boston University, McMaster University and University of Waterloo
}

\begin{abstract}
This paper examines the valuation of call options on the minimum of two dividend-paying assets. We show that the optimal exercise boundary consists of three components, two continuous curves and one component along the diagonal with empty interior. The option price is shown to satisfy the early exercise premium representation in which the gains from exercise involve the local time of the minimum of the two underlying asset prices. A system of recursive integral equations for the exercise boundary components is derived. Using a class of simple stopping times we also construct lower and upper bounds for the American call min-option price: these are easy to compute and can be employed to design efficient approximations of the contract value.
\end{abstract}

1. Introduction. Recent literature has placed much emphasis on the valuation of options that are written on multiple underlying assets [Tan and Vetzal (1995), Geltner, Riddiough and Stojanovic (1996), Broadie and Detemple (1997), Villeneuve (1999)]. Interest in these derivatives is fostered by the rapid pace of innovations that have taken place in financial markets. Contracts such as spread options (traded on the NYMEX) can now be bought in organized exchanges while options on the maximum or minimum of two or more assets are routinely quoted over-the-counter. Multiasset options are also often embedded in financial securities issued by firms or in their capital budgeting decision problems. Options on the maximum and minimum of several assets are the fundamental building blocks of more complex financial products as well as financial and capital budgeting operations engineered by firms.

Multiasset options with convex payoff functions, such as call options on the maximum of two assets, have been thoroughly studied [see Broadie and Detemple (1997)]. For these contracts the methodology employed can be summarized as a three-step process. First, the geometric structure of the exercise region is identified. Second, the value of the contract is derived in terms of the exercise boundary. Finally, the so-called smooth fit condition is established and used to characterize the exercise boundary. When the payoff is a convex function of the underlying assets standard approaches such as variational inequalities or free boundary PDE

Received August 1998; revised November 2001.

${ }^{1}$ Supported in part by a Natural Sciences and Engineering Research Council of Canada MITACS grant.

AMS 2000 subject classifications. Primary 91B28; secondary 60G40, 62L15.

Key words and phrases. Option valuation, calls, American-style, minimum of two assets, dividends, exercise premium, local time, lower and upper bounds, numerical computation. 
methods resolve the smoothness of the value function and lead to a characterization of the exercise boundary simultaneously.

However, American options with nonconvex payoffs are often required to hedge certain categories of risk exposures. Contracts of this type may be attractive to an issuer when they limit potential liabilities. From a purchaser's point of view they provide a hedging tool which might be less costly than alternative contracts. A call min-option, which pays the minimum of two or more asset prices, falls in this category since it can be viewed as a call option on one of the assets but with a stochastic cap given by another asset's price. In fact the typical capped call option is a special case of a call min-option where one of the assets has null volatility [see Broadie and Detemple $(1995,1999)]$.

In practice, min-options (or options with stochastic caps) appear in several contexts. For example, in the energy market, fuel switching technology allows a utility to choose either gas or coal as fuel fired by a power plant. In those situations it is typical for the utility to enter contractual arrangements which provide the right to buy the cheaper of the two types of fuel at a prespecified price. Counterparties can be fuel suppliers, utilities or other participants in energy markets. In exchange for this service the utility pays an up-front fee to the counterparty. The payoff to the utility is of the form $\left(S^{1} \wedge S^{2}-K\right)^{+}$, where $S^{i}$ represents the cost of fuel $i$, $i=1,2$, and $K$ is the agreed-upon exercise price. Timing optionality gives the American-style nature of this contract.

Takeover attempts are another example in which the bidder's payoff may involve the minimum of several values. In this instance the stochastic cap on the bidder's payoff results from the response of the incumbent managerial team and the possible emergence of competing bidders. Poison pills are examples of takeover defense mechanisms that increase a bidder's cost of acquisition. Asset sales are examples of managerial decisions that could eliminate synergies, thereby reducing the value of the target firm to a prospective buyer. In practice an incumbent managerial team could choose among several actions for the purpose of minimizing the value of the firm to the suitor at the time of the takeover attempt. If $S^{i}$ denotes the value of the firm associated with action $i$ and $K$ represents the acquisition cost, then the bidder's payoff is $\left(\min _{i} S^{i}-K\right)^{+}$. This min-option is American-style since the timing of the takeover attempt is endogenous.

In each of the examples above it is crucial to value the limited liability feature embedded in the min-option in order to properly assess benefits and costs and make optimal timing choices. Unfortunately, our knowledge about call min-options and related contracts is still limited. The absence of concrete results can be traced to the structure of the payoff function which fails to be convex and exhibits discontinuous first derivatives in the exercise region. This lack of smoothness of the payoff renders standard approaches based on variational inequalities or free boundary formulations of the problem difficult to implement. So far the literature has provided some limited insights about the structure of the exercise region for min-option contracts. For the case of non-dividend-paying underlying assets 
the exercise set was shown to be a subset of the diagonal [Villeneuve (1999)]. Numerical results for the case of dividend-paying assets suggest a boundary which may exhibit spikes [see Tan and Vetzal (1995) for numerical illustrations].

In this paper we consider the valuation problem for American calls which are written on the minimum of two dividend-paying assets and have finite maturities. The method that we develop is, in fact, completely general and can be used to handle more general payoff functions. Our contribution is fourfold. First, we show that the optimal exercise boundary consists of three components: two continuous curves $B_{t}^{l}\left(S^{1}\right), B_{t}^{u}\left(S^{2}\right)$ and one component along the diagonal (termed "diagonal" boundary) with lower extremity $B_{t}^{e}$ (see Figure 1). The presence of a diagonal boundary is unusual: this property is related to the fact that the exercise payoff exhibits discontinuous derivatives across the diagonal. Furthermore while immediate exercise may be optimal at a given point $(S, S)$ along the diagonal, it may also be suboptimal at all points arbitrarily close to $(S, S)$ but off the diagonal.

Our second contribution relates to the structure of the option price. In spite of the unusual geometry of the exercise set, general results of Rutkowski (1994) can be applied to establish the early exercise premium (EEP) representation of the minoption price. Accordingly, the American call min-option is the sum of a European min-option with identical maturity and strike and a premium for early exercise. However, unlike the standard case studied in the literature, the premium includes more than just the present value of the net gains (dividends net of interest costs)

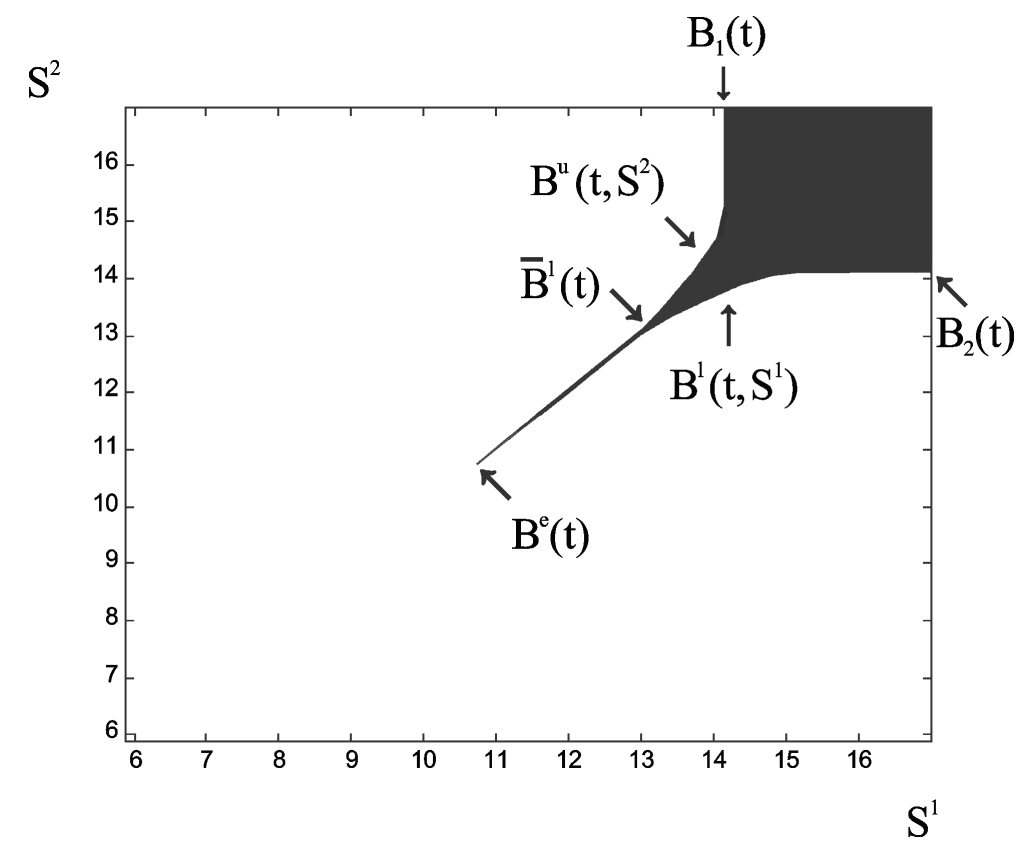

FIG. 1. Exercise region of min-option. Symmetric case: $\delta_{1}=\delta_{2}, \sigma_{1}=\sigma_{2}, \rho=0$. 
from early exercise. Due to the nonsmoothness of the payoff function when asset prices are equal (along the diagonal) there is an additional component involving the local time at zero of the difference in the underlying asset prices. The presence of this premium reflects the fact that duplication of the option payoff requires singularly continuous cash withdrawals. These occur each time the minimum of the two prices crosses the diagonal.

Third, we proceed to characterize the three boundary components. The EEP formula enables us to derive a system of coupled integral equations for the boundary components $B_{t}^{l}\left(S^{1}\right), B_{t}^{u}\left(S^{2}\right)$ and $B^{e}(t)$. Coupling follows since the exercise premium involves the gains from exercise when asset 1 has minimum price, when asset 2 has minimum price and along the diagonal when asset prices are equal, and therefore depends on all three boundary components. Our ability to characterize the lower diagonal component $B^{e}(t)$ follows from the fact that the EEP formula holds even along the diagonal. The integral equations, therefore, provide a complete characterization of all the boundary components.

Our last contribution relates to implementation. Even though the integral equations can be solved numerically by using Monte Carlo simulation, computation is time-intensive. To bypass this difficulty we develop an alternative implementable approach based on an approximation of the optimal exercise policy. Specifically, we identify a class of stopping times that can be used to construct upper and lower bounds for the call min-option value and lower bounds for the components of the exercise boundary. These stopping times involve both asset prices, yet give rise to valuation formulas that can be implemented numerically. Optimization over the class of (suboptimal) exercise policies produces a lower bound for the call minoption. It can also be used to construct lower bounds for the exercise boundary components. Combining the EEP representation with the lower bounds for the boundary produces an upper bound for the min-option value. This construction generalizes the lower bound approximation and lower-upper bound approximation methodologies in Broadie and Detemple (1996) to a multiasset contract. The bounds obtained can then be used to construct various approximations of the option value.

The results in this paper shed light on several contracts that have been examined in the previous literature. For instance, Broadie and Detemple $(1995,1997)$ provide several characterizations of American capped options. Their pricing formulas are based either on the explicit identification of the optimal exercise time or on the determination of a parsimonious representation of the boundary (reduction to a discrete set of parameters). Their resulting valuation formulas typically do not isolate the role of the local time component. Specialized to that context, our EEP representation formula shows that the premium relative to a European capped option includes the local time of the underlying asset price at the cap. Furthermore, the pricing formula does not require ex-ante knowledge of the boundary. On the contrary, it can be used to deduce a recursive equation for the optimal exercise boundary as in the case of standard options. 
Our next section resolves the valuation problem for call min-options. The construction of bounds for the contract value and the components of the exercise boundary is performed in Section 3. We state our conclusions in Section 4. All proofs are collected in the Appendix.

2. American options on the minimum of two assets. We consider derivative securities written on a pair of underlying assets. The prices of the underlying assets, $S^{1}$ and $S^{2}$, satisfy the stochastic differential equations

$$
\begin{aligned}
& d S_{t}^{1}=S_{t}^{1}\left[\left(r-\delta_{1}\right) d t+\sigma_{1} d z_{t}^{1}\right], \\
& d S_{t}^{2}=S_{t}^{2}\left[\left(r-\delta_{2}\right) d t+\sigma_{2} d z_{t}^{2}\right],
\end{aligned}
$$

where $z^{1}$ and $z^{2}$ are standard Brownian motion processes with a constant correlation $\rho$. To avoid trivial cases we assume throughout that $|\rho|<1$. Moreover, $r$ denotes the constant rate of interest, $\delta_{i} \geq 0$ is the dividend rate of asset $i$ and $\sigma_{i}$ is the volatility of the price of asset $i, i=1,2$. Here the price processes (1) and (2) are represented in their risk neutral form [see Harrison and Pliska (1981)]. Throughout the paper, $E_{t}^{*}$ will denote the expectation at time $t$ under the risk neutral measure.

An American call min-option has payoff $\left(X_{t}-K\right)^{+}$at time $t$, where $X_{t}=$ $S_{t}^{1} \wedge S_{t}^{2}$ is the minimum of the two asset prices. Let $C_{t}^{m}\left(S_{t}^{1}, S_{t}^{2}\right)$ denote the minoption value. We first review several standard facts which will be used in our subsequent analysis.

Let $F_{t}=e^{-r t}\left(X_{t}-K\right)^{+}$represent the discounted value of the payoff. Since each process $e^{-r t} S_{t}^{i}$ is a supermartingale, so is $e^{-r t} X_{t}$. Using the optional sampling theorem [Karatzas and Shreve (1987), pages 19-21] gives the following integrability property:

$$
E_{t}^{*}\left[e^{-r \tau}\left(X_{\tau}-K\right)^{+}\right] \leq \min \left(E_{t}^{*}\left[e^{-r \tau} S_{\tau}^{1}\right], E_{t}^{*}\left[e^{-r \tau} S_{\tau}^{2}\right]\right)<\infty,
$$

where $\tau$ is any stopping time in $[t, T]$. Moreover, the Doob-Meyer decomposition theorem [Karatzas and Shreve (1987), pages 24-26] implies that $e^{-r t}\left(X_{t}-K\right)$ is a continuous semimartingale. It follows from Meyer's theorem [Karatzas and Shreve (1987), Theorem 6.22, page 214] applied to $g(x)=x^{+}$that the process $F_{t}$ is a continuous semimartingale and belongs to $\mathrm{H}^{1}$ in the sense of Jacod (1979). Define the Snell envelope of the discounted payoff,

$$
Z_{t}=\operatorname{ess} \sup _{\tau \in S_{t, T}} E_{t}^{*}\left[e^{-r \tau}\left(X_{\tau}-K\right)^{+}\right]
$$

where $\delta_{t, T}$ is the set of stopping times in $[t, T]$. The theory of optimal stopping time [Fakeev (1971), Theorems 1 and 2, or El Karoui (1981)], demonstrates that $Z_{t}$ is the minimal right-continuous left-limit supermartingale which majorizes $F_{t}$ and that the optimal stopping time is

$$
\tau_{t}=\inf \left\{s \geq t: Z_{s}=F_{s}\right\} \wedge T .
$$

By a standard no-arbitrage argument [Karatzas (1988)], the rational option price is $C_{t}^{m}\left(S_{t}^{1}, S_{t}^{2}\right)=e^{r t} Z_{t}$ at all times $t \in[0, T]$. Finally, the price function $C^{m}: \mathbb{R}^{+} \times$ $\mathbb{R}^{+} \times[0, T] \rightarrow \mathbb{R}^{+}$is continuous [see Krylov (1980), Theorems 3.1.8 and 3.1.10]. 
2.1. Exercise region of American call min-options. We first identify the structure of the exercise region, $\mathcal{E}=\left\{(t, x, y) \in[0, T] \times \mathbb{R}^{+} \times \mathbb{R}^{+}: C_{t}^{m}(x, y)=\right.$ $\left.(x \wedge y-K)^{+}\right\}$of the call min-option. Define the sets

$$
\begin{aligned}
& \mathcal{E}_{t}=\left\{(x, y) \in \mathbb{R}^{+} \times \mathbb{R}^{+}: C_{t}^{m}(x, y)=(x \wedge y-K)^{+}\right\}, \\
& \mathcal{E}_{t}^{1}=\left\{(x, y) \in \mathcal{E}_{t}: x \geq y\right\}, \\
& \mathcal{E}_{t}^{2}=\left\{(x, y) \in \mathcal{E}_{t}: x \leq y\right\} .
\end{aligned}
$$

The set $\varepsilon_{t}$ represents the $t$-section of the exercise region; $\varepsilon_{t}^{i}$ is the subset of $\varepsilon_{t}$ in which asset $i$ is more expensive. Clearly $\varepsilon_{t}^{1} \cap \mathcal{E}_{t}^{2}=\left\{(x, y) \in \mathcal{E}_{t}: x=y\right\}$ and $\mathcal{E}_{t}=\mathcal{E}_{t}^{1} \cup \mathcal{E}_{t}^{2}$. Each of these sets is a closed subset of $\mathbb{R}^{+} \times \mathbb{R}^{+}$.

To describe some properties of the exercise region it is also useful to consider an option on a weighted average of assets, with payoff $(w x+(1-w) y-K)^{+}$, $w \in(0,1)$. Let $C_{t}^{w}(x, y)$ denote the option's price and $\mathcal{E}^{w}=\{(t, x, y) \in[0, T] \times$ $\left.\mathbb{R}^{+} \times \mathbb{R}^{+}: C_{t}^{w}(x, y)=(w x+(1-w) y-K)^{+}\right\}$its exercise region. The $t$-section is $\mathcal{E}_{t}^{w}$ and the exercise boundary $B_{t}^{w}(x)$. The properties of $\mathcal{E}^{w}, B_{t}^{w}(x)$ and $C_{t}^{w}(x, y)$ are detailed in Broadie and Detemple (1997).

Finally, let $B_{i}(t)$ denote the exercise boundary of a standard call option with payoff $\left(S^{i}-K\right)^{+}, i=1,2$. It has been shown that $B_{i}(t)$ solves the recursive integral equation [see Kim (1990), Jacka (1991), Carr, Jarrow and Myneni (1992)]

$$
B_{i}(t)-K=C_{i}\left(B_{i}(t), t ; B_{i}(\cdot)\right), \quad \lim _{t \rightarrow T} B_{i}(t)=K \vee \frac{r}{\delta_{i}} K
$$

where

$$
\begin{gathered}
C_{i}\left(S_{t}^{i}, t ; B_{i}(\cdot)\right)=C_{i}^{e}\left(S_{t}^{i}, t\right)+\int_{t}^{T} \phi_{i}\left(S_{t}^{i}, B_{i}(s), s-t\right) d s, \\
\phi_{i}\left(S_{t}^{i}, B_{i}, v\right) \equiv \delta_{i} S_{t}^{i} e^{-\delta_{i} v} N\left(d_{i}\left(S_{t}^{i}, B_{i}, v\right)\right)-r K e^{-r v} N\left(d_{i}\left(S_{t}^{i}, B_{i}, v\right)-\sigma_{i} \sqrt{v}\right), \\
d_{i}\left(S_{t}^{i}, B_{i}, v\right) \equiv \frac{\log \left(S_{t}^{i} / B_{i}\right)+\left(r-\delta_{i}+\frac{1}{2} \sigma_{i}^{2}\right) v}{\sigma_{i} \sqrt{v}}
\end{gathered}
$$

and $C_{i}^{e}\left(S_{t}^{i}, t\right)$ is the value of a European call option on the same underlying asset; $N(x)$ is the standard Normal distribution function.

The following properties are instrumental throughout our discussion.

PROPOSITION 1. The immediate exercise region has the following properties:

(i) If $(x, y) \in \mathcal{E}_{t}^{1}$, then $(\lambda x, \lambda y) \in \mathcal{E}_{t}^{1}$ for all $\lambda \geq 1$.

(ii) If $(x, y) \in \mathcal{E}_{t}^{1}$, then $(x, \lambda y) \in \mathcal{E}_{t}^{1}$ for all $1 \leq \lambda \leq x / y$.

(iii) If $(x, y) \in \mathcal{E}_{t}{ }_{t}$, then $(\lambda x, y) \in \mathcal{E}_{t}{ }_{t}^{1}$ for all $y / x \leq \lambda \leq 1$. 
(iv) If $(x, y) \in \mathbb{R}^{+} \times \mathbb{R}^{+}$and $x \geq y \geq B_{2}(t)$, then $(x, y) \in \mathcal{E}_{t}^{1}$.

(v) For any $w \in(0,1)$, if $(x, y) \in \mathcal{E}_{t}^{w}$ and $x=y$, then $(x, y) \in \mathcal{E}_{t}^{1}$.

Results similar to (i)-(v) also hold for $\mathcal{E}_{t}^{2}$. Finally, we have the following:

(vi) $C_{t}^{m}(x, y)$ is a nondecreasing function of $x, y$ and is a nonincreasing function of time $t$.

Property (i) states that the exercise subregion $\varepsilon_{t}^{1}$ is ray-connected. It is also upconnected [property (ii)] and left-connected [property (iii)] until the diagonal is reached. Property (iv) means that immediate exercise of a min-option is optimal if immediate exercise of an option on asset 2 alone is optimal: this is intuitively evident since the payoff of the min-option is bounded above by the payoffs of the single asset options.

Property (v) establishes an interesting connection between the weighted average option and the min-option. Indeed, note that the min-option payoff is always bounded above by the payoff of any weighted average option. This means that the price of the average option is an upper bound for the price of the min-option. Furthermore, along the diagonal the two payoffs coincide [i.e., $(x \wedge y-K)^{+}=$ $\left.(w x+(1-w) y-K)^{+}=(x-K)^{+}\right]$. It follows that immediate exercise must be optimal for the min-option if immediate exercise is optimal for the weighted average option. As we shall see this provides an upper bound for the exercise boundary of the min-option along the diagonal.

Finally, property (vi) states that the option cannot lose value when either of the underlying prices increases or time to maturity increases.

Since the function $C_{t}^{m}(x, y)$ is continuous and the sets $\varepsilon_{t}, \varepsilon_{t}^{1}, \varepsilon_{t}^{2}$ are closed we can define the sections

$$
\begin{aligned}
B_{t}^{l}(x) & =\inf \left\{y>0: y \leq x,(x, y) \in \mathcal{E}_{t}^{1}\right\}, \\
B_{t}^{u}(y) & =\inf \left\{x>0: y \geq x,(x, y) \in \mathcal{E}_{t}^{2}\right\}, \\
B_{t}^{e} & =\inf \left\{x>0: B_{t}^{l}(x)=x\right\} .
\end{aligned}
$$

Clearly, $B_{t}^{e}=\inf \left\{y>0: B_{t}^{u}(y)=y\right\}$. The function $B_{t}^{l}(x)$ [resp. $\left.B_{t}^{u}(y)\right]$ represents the boundary of the exercise set at date $t$ in the region $\mathcal{E}_{t}^{1}$ (resp. $\mathcal{E}_{t}^{2}$ ). These two boundaries meet and merge along the diagonal (see Figure 1). The point $B_{t}^{e}$ represents the lowest value taken by either boundary on the diagonal. Our next proposition establishes key properties of the exercise boundaries.

Proposition 2. The boundary $B_{t}^{l}(x)$ has the following properties, for all $t \in[0, T]:$

(i) $B_{t}^{l}(x)$ is a nondecreasing function of $x$;

(ii) if $B_{t}^{l}\left(x_{i}\right)=x_{i}$ for $x_{1}<x_{2}$, then $B_{t}^{l}(x)=x$ for all $x \in\left[x_{1}, x_{2}\right]$; 
(iii) $B_{t}^{e}=\inf \left\{x>0: B_{t}^{l}(x)=x\right\} \leq B_{t}^{w}\left(x^{*}\right)$, where $x^{*}$ uniquely solves $B_{t}^{w}(x)=x$;

(iv) $B_{t}^{l}(x)$ is absolutely continuous and differentiable almost everywhere;

(v) $\lim _{t \uparrow T} B_{t}^{l}(x)=K \vee \frac{r}{\delta_{2}} K$ if $B_{t}^{l}(x)<x$ and $\lim _{t \uparrow T} B_{t}^{e}=K$;

(vi) the bivariate function $B^{l}:(t, x) \rightarrow B_{t}^{l}(x)$ is continuous.

Similar results hold for $B_{t}^{u}(y)$.

Property (i) implies that the critical exercise levels cannot decrease as asset prices increase. Property (ii) states that the exercise boundary is a connected set along the diagonal: if there exist two boundary points on the diagonal, then any point in between also belongs to the boundary. This foreshadows a striking result, namely that the exercise set may have a connected component, with empty interior, along the diagonal. The possibility of this unusual feature of the min-option is in sharp contrast with standard cases and deserves further discussion.

Some intuition for the possible optimality of exercise along the diagonal, when it is suboptimal to exercise off the diagonal, can be provided as follows. To simplify matters assume that prices are independent. Consider a diagonal point and suppose that the option holder forgoes exercise at that point. Note that the probability of an increase in any one of the underlying asset prices, over the next small increment of time $h$, is approximately equal to $\frac{1}{2}$. Thus, the probability of an increase in the minimum of the two asset prices is roughly equal to $\frac{1}{4}$ over $h$, which implies that the expected local benefits of any waiting policy are negative. This intuition is exactly the opposite of the one for a call option on the maximum of two asset prices. Recall that immediate exercise, of a max-call, along the diagonal is suboptimal at any time prior to maturity [see Broadie and Detemple (1997)]. This follows because the max-call payoff has a probability roughly equal to $\frac{3}{4}$ of increasing over the next increment of time, which induces a positive expected local benefit of waiting along the diagonal.

Properties (i) and (ii) enable us to define the (time-dependent) points

$$
\begin{aligned}
\bar{B}_{t}^{l} & =\sup \left\{x: B_{t}^{l}(x)=x\right\}, \\
\bar{B}_{t}^{u} & =\sup \left\{y: B_{t}^{u}(y)=y\right\} .
\end{aligned}
$$

Moreover, we see that the curve $y=B_{t}^{l}(x)$ starts from the point $\left(B_{t}^{e}, B_{t}^{e}\right)$, follows the diagonal up to the point $\left(\bar{B}_{t}^{l}, \bar{B}_{t}^{l}\right)$ after which it branches off and falls (strictly) below the diagonal. In fact, when $x \rightarrow \infty, B_{t}^{l}(x) \rightarrow B_{2}(t)$. Similarly, the curve $x=B_{t}^{u}(y)$ starts from $\left(B_{t}^{e}, B_{t}^{e}\right)$, follows the diagonal up to $\left(\bar{B}_{t}^{u}, \bar{B}_{t}^{u}\right)$ and then branches off (strictly) above the diagonal. As explained above the value $B_{t}^{e}$ may be smaller than both $\bar{B}_{t}^{l}$ and $\bar{B}_{t}^{u}$. That is, there may be a segment on the diagonal in the exercise region which coincides with the curves $y=B_{t}^{l}(x)$ and $x=B_{t}^{u}(y)$. This feature is also reminiscent of a property displayed by American capped options with growing caps [see Broadie and Detemple (1995)]. 
Property (iii) establishes that the lowest point of the boundary on the diagonal $B_{t}^{e}$ is located below the point $B_{t}^{w}\left(x^{*}\right)=x^{*}$ at which the boundary of the average option $B_{t}^{w}(x)$ crosses the diagonal. This property follows immediately from Proposition 1(v). This bound on $B_{t}^{e}$ could prove useful in numerical implementations of our valuation formulas.

Property (v) reflects the standard result that the exercise boundary converges at maturity to the maximum of the strike and the strike multiplied by the interest rate over the dividend yield [see (4)]. When asset 2 is the cheapest its dividend yield determines this limiting condition. When the two prices are the same the diagonal boundary component converges to the strike: this parallels a result by Villeneuve (1999) for non-dividend-paying assets.

Properties (iv) and (vi) establish the following regularity of the $t$-section of the exercise region and the continuation region. Given two open sets $U, V \subset \mathbb{R}^{+} \times \mathbb{R}^{+}$, write $U \simeq V$ if $U \cap\{(x, y): x \neq y\}=V \cap\{(x, y): x \neq y\}$, that is, $U=V$ off the diagonal. $A^{0}$ denotes the interior of any set $A$. Then it is easy to see, from the graph of the exercise region, that for any $t \in[0, T]$ we have $\overline{\mathcal{E}_{t}^{0}} \simeq \mathcal{E}_{t}$ and $\overline{\mathcal{C}_{t}^{0}} \simeq C_{t}$. This property is also proved by Villeneuve (1999) for claims with convex payoffs and infinite maturities, written on non-dividend-paying assets.

2.2. The early exercise premium representation. We now establish the early exercise premium representation for the min-option contract. For this option the general representation formula of Rutkowski (1994) applies. However, unlike typical cases studied in the literature [see Kim (1990), Jacka (1991), Carr, Jarrow and Myneni (1992), Jamshidian (1992)] the early exercise premium for the minoption involves an unusual component unrelated to dividend payments collected or interest costs incurred upon exercise. This term, which is identified below, involves a local time component which arises in the decomposition of the option's discounted payoff process.

Since $F_{t}$ is a continuous semimartingale of class $\mathrm{H}^{1}$, it admits the decomposition $F_{t}=X_{0}+M_{t}+V_{t}$, where $M$ stands for a continuous martingale, and $V$ for a continuous process of integrable variation. Moreover, we prove (see the Appendix)

$$
\begin{aligned}
d V_{t}= & \chi_{\left\{X_{t}>K\right\}} e^{-r t} r K d t+\frac{1}{2} e^{-r t} d L(t, 0 ; X-K) \\
& -\chi_{\left\{X_{t}>K\right\}} e^{-r t}\left[\delta_{1} S_{t}^{1} \chi_{\left\{S_{t}^{1}<S_{t}^{2}\right\}}+\delta_{2} S_{t}^{2} \chi_{\left\{S_{t}^{1}>S_{t}^{2}\right\}}\right] d t \\
& -\frac{1}{2}\left(\sigma_{1}^{2}-2 \sigma_{1} \rho \sigma_{2}+\sigma_{2}^{2}\right) \chi_{\left\{X_{t}>K\right\}} e^{-r t} X_{t} d L\left(t, 0 ; \ln \left(S^{1}\right)-\ln \left(S^{2}\right)\right),
\end{aligned}
$$

where $L(t, 0 ; X-K)$ is the local time of $X-K$ at $0, L\left(t, 0 ; \ln \left(S^{1}\right)-\right.$ $\left.\ln \left(S^{2}\right)\right)$ denotes the local time of $\ln \left(S^{1}\right)-\ln \left(S^{2}\right)$ at 0 and the symbol $\chi_{A}$ is the characteristic function of the set $A$. The local time components in the decomposition of the option's payoff are due to the discontinuities in the derivative of the payoff along the diagonal $S^{1}=S^{2}$ and at the money $X_{t}=K$. Therefore, by 
Rutkowski [(1994), Proposition A.1], we obtain

$$
E F_{\tau_{t}}=E F_{T}-E\left[\int_{\tau_{t}}^{T} I_{\left\{\tau_{u}=u\right\}} d V_{u}\right] .
$$

A consequence is the following early exercise premium representation of the price function.

THEOREM 3. The price of the call min-option has the representation

$$
C_{t}^{m}\left(S_{t}^{1}, S_{t}^{2}\right)=C_{t}^{e}\left(S_{t}^{1}, S_{t}^{2}\right)+\Pi_{t}\left(S_{t}^{1}, S_{t}^{2} ; B^{l}, B^{u}, B^{e}\right),
$$

where $C_{t}^{e}\left(S_{t}^{1}, S_{t}^{2}\right)$ is the price of the European min-option and $\Pi_{t}\left(S_{t}^{1}, S_{t}^{2} ; B^{l}, B^{u}\right.$, $\left.B^{e}\right)=\Pi_{1}+\Pi_{2}+\Pi_{3}$ is the early exercise premium with

$$
\begin{aligned}
\Pi_{1}= & E_{t}^{*}\left[\int_{t}^{T} e^{-r(v-t)}\left(\delta_{1} S_{v}^{1}-r K\right) \chi_{\left\{B_{v}^{u}\left(S_{v}^{2}\right) \leq S_{v}^{1}<S_{v}^{2}\right\}} d v\right], \\
\Pi_{2}= & E_{t}^{*}\left[\int_{t}^{T} e^{-r(v-t)}\left(\delta_{2} S_{v}^{2}-r K\right) \chi_{\left\{B_{v}^{l}\left(S_{v}^{1}\right) \leq S_{v}^{2}<S_{v}^{1}\right\}} d v\right], \\
\Pi_{3}= & \frac{1}{2}\left(\sigma_{1}^{2}-2 \sigma_{1} \rho \sigma_{2}+\sigma_{2}^{2}\right) E_{t}^{*} \\
& \times\left[\int_{t}^{T} e^{-r(v-t)} \chi_{\left\{S_{v}^{1}=S_{v}^{2} \geq B_{v}^{e}\right\}} X_{v} d L\left(v, 0 ; \ln \left(S^{1}\right)-\ln \left(S^{2}\right)\right)\right] .
\end{aligned}
$$

The EEP representation decomposes the price of the American min-option into two parts. The first is the value of a European min-option with identical characteristics (maturity date and strike). The second is a premium which captures the additional value created by the possibility of optimal early exercise. Unlike standard cases, the exercise premium is the sum of two distinct parts. One is the present value of the net gains (dividends net of interest costs) from early exercise, $\Pi_{1}+\Pi_{2}$. The dividend benefits embedded in these terms are collected on asset 1 (resp. asset 2) when $S^{1}$ (resp. $S^{2}$ ) is the minimum of the two asset prices.

The other part, $\Pi_{3}$, is related to the lack of smoothness of the payoff function along the diagonal, in the exercise region. Each time the minimum of the two asset prices crosses the diagonal or the strike the derivatives of the payoff function jump. In effect the local time processes $L\left(t, 0 ; \ln \left(S^{1}\right)-\ln \left(S^{2}\right)\right)$ and $L(t, 0 ; X-K)$ compensate for these jumps. From a financial point of view the presence of the local time components indicates that the option payoff cannot be duplicated by a self-financing portfolio of the riskless asset and the two stocks. For this payoff, duplication requires cumulative cash withdrawals given by

$$
\frac{1}{2}\left(\sigma_{1}^{2}-2 \sigma_{1} \rho \sigma_{2}+\sigma_{2}^{2}\right) \int_{0}^{t} \chi_{\left\{X_{v}>K\right\}} e^{-r v} X_{v} d L\left(v, 0 ; \ln \left(S^{1}\right)-\ln \left(S^{2}\right)\right)
$$

to account for the behavior on the diagonal and cumulative cash infusions

$$
\frac{1}{2} \int_{0}^{t} e^{-r v} d L(v, 0 ; X-K)
$$


to account for the behavior at the money (i.e., when $X=K$ ). Naturally, since early exercise will never take place at the money, only the first component will affect the option value.

The expression for $\Pi_{3}$ sheds further light on the role of the diagonal boundary component. First note that $\sigma_{1}^{2}-2 \sigma_{1} \rho \sigma_{2}+\sigma_{2}^{2}>0$, except when assets are perfectly correlated and equally risky (i.e., $\sigma_{1}=\sigma_{2}$ and $\rho=1$ ). In that situation asset prices are related by $S_{t}^{2}=S_{t}^{1}\left(S_{0}^{2} / S_{0}^{1}\right) \exp \left(\left(\delta_{1}-\delta_{2}\right) t\right)$ and the min-option reduces to a call option on a single underlying asset with a time-dependent quantity adjustment [i.e., the option's payoff is $\left(Q(t) S_{t}^{1}-K\right)^{+}$, where $Q(t)=1 \wedge\left(S_{0}^{2} / S_{0}^{1}\right) \exp \left(\left(\delta_{1}-\delta_{2}\right) t\right]$. Naturally, the local time component in the exercise premium vanishes since the payoff is smooth with respect to the single underlying Brownian motion.

Second, if $\sigma_{1}=0$ or $\sigma_{2}=0$, the min-option reduces to a capped call option with time-dependent cap. This class of contracts was studied by Broadie and Detemple (1995, 1997, 1999), who established two representations of the price. The first one expresses the price as the present value of the payoff at the exercise date, which is identified explicitly using dominance arguments. The second applies to the constant cap case and decomposes the price as the value of an option with automatic exercise at the cap plus the benefits from exercising prior to reaching the cap. Although the latter representation is an EEP-style formula it does not involve a local time component since possible payoffs are computed before the underlying asset price crosses the cap. Thus, the formula of Theorem 3 provides a new decomposition of capped option prices. It identifies the benefits from early exercise relative to a European capped option (where the payoff accrues at $T$ ) and shows that these benefits include a local time gain component.

Finally, in the general case when $\sigma_{1} \sigma_{2} \neq 0$ and $\rho \neq 1$, the premium $\Pi_{3}>0$. In this instance the exercise boundary is described by the three components $B_{t}^{l}\left(S^{1}\right), B_{t}^{u}\left(S^{2}\right)$ and $B^{e}(t)$. In the particular case of non-dividend-paying assets $\left(\delta_{1}=\delta_{2}=0\right)$ the premia $\Pi_{1}, \Pi_{2}$ vanish. It is straightforward to see that the boundary curves $B_{t}^{l}\left(S^{1}\right), B_{t}^{u}\left(S^{2}\right)$ collapse to the diagonal (i.e., $\bar{B}_{t}^{l}, \bar{B}_{t}^{u} \rightarrow \infty$ ) and that the exercise region reduces to the diagonal segment above $B^{e}(t)$ (see details in the next section). This fact was proved by Villeneuve (1999) using a different method.

REMARK 1. An explicit formula for the European price function is provided by Johnson (1987). This formula shows that $C_{t}^{e}\left(S_{t}^{1}, S_{t}^{2}\right)$ is a smooth function. Similar expressions can also be derived for the premium components $\Pi_{1}$ and $\Pi_{2}$.

The EEP representation provides an intuitive financial interpretation of the sources of value embedded in the contract. In addition it can be employed to establish key properties of the price function (see Proposition 9 in the Appendix for details). However, its most interesting use is perhaps the fact that it leads to a characterization of the exercise boundary which has practical importance. This is the issue discussed next. 
2.3. The integral equation for the boundary. The EEP representation of the American option price is valid at any point in the state space, and in particular in the exercise region. Since immediate exercise is optimal in this region we deduce the following integral equation characterization of the exercise boundary.

THEOREM 4. The immediate exercise boundary $\left(B_{t}^{l}(x), B_{t}^{u}(y), B_{t}^{e}\right)$ solves the triplet of integral equations

$$
\begin{aligned}
B_{t}^{l}(x)-K & =C_{t}^{e}\left(x, B_{t}^{l}(x)\right)+\Pi_{t}\left(x, B_{t}^{l}(x) ; B^{l}, B^{u}, B^{e}\right), \\
B_{t}^{u}(y)-K & =C_{t}^{e}\left(B_{t}^{u}(y), y\right)+\Pi_{t}\left(B_{t}^{u}(y), y ; B^{l}, B^{u}, B^{e}\right), \\
B_{t}^{e}-K & =C_{t}^{e}\left(B_{t}^{e}, B_{t}^{e}\right)+\Pi_{t}\left(B_{t}^{e}, B_{t}^{e} ; B^{l}, B^{u}, B^{e}\right)
\end{aligned}
$$

subject to the boundary conditions $\lim _{t \rightarrow T} B_{t}^{l}(x)=K \vee r K / \delta_{2}$ for $B_{t}^{l}(x)<x$, $\lim _{t \rightarrow T} B_{t}^{u}(y)=K \vee r K / \delta_{1}$ for $B_{t}^{u}(y)<y$ and $\lim _{t \rightarrow T} B_{t}^{e}=K$. The solution is unique if Condition $\mathrm{A}$ or Condition $\mathrm{B}$ in the Appendix holds.

This theorem shows that the boundary components $B_{t}^{l}(x)$ and $B_{t}^{u}(y)$ satisfy a system of coupled equations. Coupling follows from the structure of the exercise premium which depends on both boundary surfaces. Note that the last equation is redundant: the curve $B_{t}^{e}$ could simply be recovered by taking the lowest value of $B_{t}^{l}(x)$ [or $B_{t}^{u}(y)$ ] along the diagonal.

In summary the recursive equations of Theorem 4 provide a complete characterization of all the boundary components. These equations can be used in a numerical scheme to compute an approximation of the boundary and, hence, of the option value. Computation can be performed by Monte Carlo simulation. The price-dependence of the boundary components, however, implies that computation is expensive. This motivates the search for alternative approaches that are easier to implement.

3. Simple bounds for call min-options. The main purpose of this section is to provide upper and lower bounds for American call min-option prices that are implementable in numerical applications and provide reasonable estimates of value. Our approach is based on the identification of a class of suboptimal stopping times that approximate the optimal exercise policy and can be valued. The results reported below extend the method of Broadie and Detemple (1996) to call minoptions on multiple assets. We first derive a lower bound (Section 3.1) and an upper bound (Section 3.2) for the contract value. A one-dimensional lower bound for the boundary and an associated price upper bound are constructed next (Section 3.3). A numerical example concludes the section (Section 3.4).

3.1. A lower bound for the call min-option price (lower bound approximation). The main ingredient, leading to the construction of a lower bound, is the 
identification of a class of exercise policies leading to computable valuation formulas. One particularly simple class of stopping times, which seems promising in that regard, consists of barrier policies which mandate exercise once the underlying asset prices have breached constant barriers.

Let us then consider two constant barriers $L^{1}, L^{2}>K$ and such that $S^{1} \leq L^{1}$, $S^{2} \leq L^{2}$. Suppose that the option holder adopts the exercise policy represented by the stopping time $\tau=\tilde{\tau} \wedge T$, where

$$
\tilde{\tau}=\inf \left\{s \in[t, T]: S_{s}^{1} \geq L^{1} \text { and } S_{s}^{2} \geq L^{2}\right\}
$$

or $\tilde{\tau}=\infty$ if no such time exists in $[t, T]$. Under this policy exercise takes place when the underlying prices simultaneously exceed their respective barriers, or at the maturity date if that event does not occur prior to maturity. The value of the barrier policy $\tau$ is given by

$$
C_{t}\left(S^{1}, S^{2} ; L^{1}, L^{2}\right)=\hat{C}_{1}(t)+\hat{C}_{2}(t)
$$

where

$$
\begin{aligned}
& \hat{C}_{1}(t) \equiv E_{t}^{*}\left[e^{-r(\tilde{\tau}-t)}\left(S_{\tilde{\tau}}^{1} \wedge S_{\tilde{\tau}}^{2}-K\right)^{+} \chi_{\{\tilde{\tau} \leq T\}}\right], \\
& \hat{C}_{2}(t) \equiv E_{t}^{*}\left[e^{-r(T-t)}\left(S_{T}^{1} \wedge S_{T}^{2}-K\right)^{+} \chi_{\{\tilde{\tau}=\infty\}}\right] .
\end{aligned}
$$

The first term, $\hat{C}_{1}$, is the value of the cash flows collected if $\{\tilde{\tau} \leq T\}$; the second component, $\hat{C}_{2}$, is the value derived from exercising at maturity, in the event $\{\tilde{\tau}=\infty\}$.

The value $C_{t}\left(S^{1}, S^{2} ; L^{1}, L^{2}\right)$ is a lower bound for the American min-option call price since the policy of exercising at $\tau$ is feasible for the holder of the min-option. Moreover, maximization over the pair of constant barriers $\left(L^{1}, L^{2}\right)$ produces the best lower bound in the class of exercise policies under consideration. Thus, taking the supremum over $L^{1}, L^{2}$ gives the function

$$
C_{t}^{l}\left(S^{1}, S^{2}\right) \equiv \sup _{L^{1} \geq S^{1}, L^{2} \geq S^{2}} C_{t}\left(S^{1}, S^{2} ; L^{1}, L^{2}\right),
$$

which is a lower bound approximation (LBA) of the American min-call option value. This LBA improves on the European min-option since $C_{t}\left(S^{1}, S^{2} ; L^{1}\right.$, $\left.L^{2}\right) \rightarrow C_{t}^{e}\left(S^{1}, S^{2}\right)$ as $L^{1}, L^{2} \uparrow \infty$. It also improves on the immediate exercise payoff which is obtained by choosing $L^{i}=S^{i}, i=1,2$.

In implementations the price $C_{t}\left(S^{1}, S^{2} ; L^{1}, L^{2}\right)$, parametrized by the pair of constants $\left(L^{1}, L^{2}\right)$, can be easily computed using Monte Carlo simulation. The optimization problem embedded in the lower bound $C_{t}^{l}\left(S^{1}, S^{2}\right)$ can be resolved using a grid search algorithm.

3.2. An upper bound for the call min-option price (upper bound approximation). To derive an upper bound for the option price we first construct a pair of 
lower bounds $\left(L_{t}^{l}(\cdot), L_{t}^{u}(\cdot)\right)$ for the exercise boundary components $\left(B_{t}^{l}(\cdot), B_{t}^{u}(\cdot)\right)$. This construction is based on the class of lower bounds $C_{t}\left(S^{1}, S^{2} ; L^{1}, L^{2}\right)$ for the option price discussed above. Combining the lower bounds for the boundary components with the EEP formula produces an upper bound for the option price.

Let us first outline the construction of a lower bound $L_{t}^{l}(\cdot)$ for the curve $B_{t}^{l}(\cdot)$. The same procedure applies to $B_{t}^{u}(\cdot)$.

Pick a point $\left(S^{1}, S^{2}\right)$ such that $S^{2}=B_{2}(t) \wedge S^{1}$ [i.e., the point $\left(S^{1}, S^{2}\right)$ is either on the diagonal or on the line $\left.B_{2}(t)\right]$. For constants $L^{1} \geq S^{1}$ and $L^{2} \geq S^{2}$ define the new lower bound for the min-option price

$$
\left.C_{t}^{2}\left(S^{1}, S^{2} ; L^{2}\right) \equiv C_{t}\left(S^{1}, S^{2} ; L^{1}, L^{2}\right)\right|_{L^{1}=S^{1}}=C_{t}\left(S^{1}, S^{2} ; S^{1}, L^{2}\right) .
$$

Now select $\varepsilon>0$ and construct a sequence $L^{2, n}\left(S^{1}\right)$ by solving the univariate optimization problem

$$
L^{2, n}\left(S^{1}\right)=\arg \max _{L}\left\{C_{t}^{2}\left(S^{1}, L^{2, n-1}\left(S^{1}\right)-\varepsilon ; L\right): L \geq L^{2, n-1}\left(S^{1}\right)-\varepsilon\right\}
$$

for $n=1,2, \ldots$, with initial condition $L^{2,0}=S^{2}$. Note that the solution of this problem is $L^{2, n}\left(S^{1}\right)=L^{2, n-1}\left(S^{1}\right)-\varepsilon$ as long as $\left(S^{1}, L^{2, n-1}\left(S^{1}\right)-\varepsilon\right)$ is in the exercise region of the min-option. Indeed, suppose that $\left(S^{1}, L^{2, n-1}\left(S^{1}\right)-\varepsilon\right) \in \varepsilon_{t}^{1}$. Then

$$
\begin{aligned}
L^{2, n-1}\left(S^{1}\right)-\varepsilon-K & =C_{t}^{m}\left(S^{1}, L^{2, n-1}\left(S^{1}\right)-\varepsilon\right) \\
& \geq \sup _{L \geq L^{2, n-1}\left(S^{1}\right)-\varepsilon} C_{t}^{2}\left(S^{1}, L^{2, n-1}\left(S^{1}\right)-\varepsilon ; L\right) \\
& \geq L^{2, n-1}\left(S^{1}\right)-\varepsilon-K,
\end{aligned}
$$

where the last inequality follows from the fact that immediate exercise is feasible for $C_{t}^{2}$ [set $L=L^{2, n-1}\left(S^{1}\right)-\varepsilon$ ] and achieves the payoff $L^{2, n-1}\left(S^{1}\right)-\varepsilon-K$. Thus, by construction the sequence $\left(L^{2, n}\left(S^{1}\right)\right)$ is strictly decreasing as long as the lagged sequence $\left(L^{2, n-1}\left(S^{1}\right)-\varepsilon\right) \in\left[B^{l}\left(t, S^{1}\right), B_{2}(t)\right)$. Set

$$
n^{\varepsilon}\left(S^{1}\right)=\inf \left\{n: L^{2, n}\left(S^{1}\right)>L^{2, n-1}\left(S^{1}\right)-\varepsilon\right\}
$$

and define $\left.L^{2 \varepsilon}\left(S^{1}\right) \equiv L^{2, n-1}\left(S^{1}\right)\right|_{n=n^{\varepsilon}\left(S^{1}\right)}-\varepsilon$. By construction $L^{2 \varepsilon}\left(S^{1}\right)$ is a lower bound for $B_{t}^{l}\left(S^{1}\right)$ at the point $S^{1}$. Letting $\varepsilon \rightarrow 0$ gives a lower bound $L^{l}\left(S^{1}\right)$.

Denote by $L^{u}\left(S^{2}\right)$ the solution of the equation obtained by the symmetric construction. We have the following proposition.

Proposition 5. For each pair $\left(t, S^{1}\right)$, we have $L_{t}^{l}\left(S_{t}^{1}\right) \leq B_{t}^{l}\left(S_{t}^{1}\right)$. Symmetrically, for each $\left(t, S^{2}\right), L_{t}^{u}\left(S_{t}^{2}\right) \leq B_{t}^{u}\left(S_{t}^{2}\right)$.

Consider the early exercise premium representation of the American call minoption, in Theorem 3, where we replace the boundary functions $B_{t}^{l}\left(S_{t}^{1}\right), B_{t}^{u}\left(S_{t}^{2}\right)$, $B_{t}^{e}$ by $L_{t}^{l}\left(S_{t}^{1}\right), L_{t}^{u}\left(S_{t}^{2}\right), L_{t}^{e}$, respectively $\left(L_{t}^{e}\right.$ is the smallest value, obtained from 
the algorithm above, along the diagonal). This produces a new function, denoted by $C_{t}^{u}\left(S_{t}^{1}, S_{t}^{2}\right)$, parametrized by the curves $L_{t}^{l}\left(S_{t}^{1}\right), L_{t}^{u}\left(S_{t}^{2}\right), L_{t}^{e}$. Our next result establishes that this function is an upper bound for the call min-option value. The proof follows straightforwardly from the EEP representation in Theorem 3, from the bounds $L_{t}^{l}\left(S_{t}^{1}\right) \geq K \vee r K / \delta_{2}$ and $L_{t}^{u}\left(S_{t}^{2}\right) \geq K \vee r K / \delta_{1}$ off the diagonal and $L_{t}^{e} \geq K$ along the diagonal and from Proposition 5. Combining this result with those of the previous subsection gives the following theorem.

THEOREM 6. The American call min-option value function $C^{m}\left(S^{1}, S^{2}, T-t\right)$ has the lower and upper bounds $C_{t}^{l}\left(S^{1}, S^{2}\right) \leq C^{m}\left(S^{1}, S^{2}, T-t\right) \leq C_{t}^{u}\left(S^{1}, S^{2}\right)$ for all $\left(t, S^{1}, S^{2}\right) \in \mathcal{C}$.

It is easily shown that the bounds in Theorem 6 are tight for options approaching maturity, for deep out-of-the money and for deep in-the-money options. Similar results can also be proved for extreme low and high volatilities, large dividend rates and large interest rates.

The numerical computation of $L_{t}^{l}\left(S_{t}^{1}\right), L_{t}^{u}\left(S_{t}^{2}\right), L_{t}^{e}$ and the price bound $C_{t}^{u}\left(S_{t}^{1}, S_{t}^{2}\right)$ can again be performed using Monte Carlo simulation. Computation is expected to be time-consuming. Efficiency can be improved by constructing lower bounds for the exercise boundaries that are less computationally intensive. In fact, any set of functions $H_{t}^{l}\left(S_{t}^{1}\right), H_{t}^{u}\left(S_{t}^{2}\right), H_{t}^{e}$ such that $K \vee r K / \delta_{2} \leq H_{t}^{l}\left(S_{t}^{1}\right) \leq B_{t}^{l}\left(S_{t}^{1}\right)$ for $H_{t}^{l}\left(S_{t}^{1}\right)<S_{t}^{1}$ and $K \vee r K / \delta_{1} \leq H_{t}^{u}\left(S_{t}^{2}\right) \leq B_{t}^{u}\left(S_{t}^{2}\right)$ for $H_{t}^{u}\left(S_{t}^{2}\right)<S_{t}^{2}$ and $K \leq H_{t}^{e} \leq B_{t}^{e}$ can be substituted in the EEP representation to produce an upper bound for the price. A simple triplet that does not require any computation is $\left(K \vee r K / \delta_{1}, K \vee r K / \delta_{2}, K\right)$. Unfortunately, the associated upper bound proved to be loose in numerical experiments. Next, we propose another pair which produces a tighter price upper bound.

3.3. A one-dimensional lower bound for the exercise boundary. The idea is to construct a time-dependent lower bound $H_{t}^{e}$ such that $K \leq H_{t}^{e} \leq B_{t}^{l}\left(S_{t}^{1}\right)$ and $K \leq H_{t}^{e} \leq B_{t}^{u}\left(S_{t}^{2}\right)$. A price upper bound is then obtained by substituting $H^{e} \vee r K / \delta_{i}$ in $\Pi_{i}, i=1,2$, and $H^{e}$ in $\Pi_{3}$.

The time-dependent lower bound $H^{e}$ can be derived by solving the problem

$$
H_{t}^{e}=\inf \left\{H: H-K=C_{t}^{e}(H, H)+\Pi_{3}(H, H ; H)\right\}
$$

for each $t \in[0, T]$. The first term on the right-hand side of (9) is the European min-option price $C_{t}^{e}(H, H)$ evaluated at $S_{t}^{1}=S_{t}^{2}=H$. The second term,

$$
\begin{aligned}
\Pi_{3}(H, H ; H)= & \frac{1}{2}\left(\sigma_{1}^{2}-2 \sigma_{1} \rho \sigma_{2}+\sigma_{2}^{2}\right) \\
& \times E_{t}^{*}\left[\int_{t}^{T} e^{-r(v-t)} \chi_{\left\{S_{v}^{1}=S_{v}^{2} \geq H\right\}} X_{v} d L\left(v, 0 ; \ln \left(S^{1}\right)-\ln \left(S^{2}\right)\right)\right],
\end{aligned}
$$

is a premium associated with the local time component also evaluated at $S_{t}^{1}=$ $S_{t}^{2}=H$ and where the indicator function is over the event $\left\{S_{v}^{1}=S_{v}^{2} \geq H\right\}$. The 
right-hand side corresponds to the value of a min-option with automatic exercise along the diagonal in the event that prices exceed the constant $H$. This value is clearly a lower bound for the American min-option. It follows immediately that $H_{t}^{e} \leq B_{t}^{e}$, for all $t \in[0, T]$. Since both value components are positive it also follows that $K \leq H_{t}^{e}$, for all $t \in[0, T]$. Finally, the definition of $B_{t}^{e}$ implies $K \leq H_{t}^{e} \leq B_{t}^{l}\left(S_{t}^{1}\right)$ and $K \leq H_{t}^{e} \leq B_{t}^{u}\left(S_{t}^{u}\right)$.

The bound $H_{t}^{e}$ is one-dimensional since it does not depend on the prices, but only on time. It can be computed by simulating the prices and the local time process and by using an iterative procedure to solve for the fixed point in (9).

3.4. A numerical example. To illustrate the results and assess the quality of the approximations we compute the min-call value when $K=100, r=6 \%$, $\delta_{1}=\delta_{2}=5 \%, \rho=0, \sigma_{1}=\sigma_{2}=0.20, T=1$ and for various combinations of $\left(S_{1}, S_{2}\right)$. Table 1 displays the European option price, the American price based on a binomial approximation, a lower bound approximation, an upper

TABLE 1

Prices and bounds for call min-option*

\begin{tabular}{|c|c|c|c|c|c|c|}
\hline$S_{2}$ & $S_{1}$ & $\begin{array}{c}\text { European } \\
\text { min-call }\end{array}$ & $\begin{array}{l}\text { Binomial } \\
\text { value } \\
(100 \text { steps })\end{array}$ & $\begin{array}{l}\text { Lower } \\
\text { bound } \\
\text { (LBA) }\end{array}$ & $\begin{array}{l}\text { Upper } \\
\text { bound } \\
\text { (UBA) }\end{array}$ & $\begin{array}{c}\text { Lower-upper } \\
\text { bound approx. (LUBA), } \\
\lambda=0.5\end{array}$ \\
\hline \multirow[t]{6}{*}{90} & 90 & 0.56 & 0.82 & 0.63 & 0.97 & 0.80 \\
\hline & 100 & 1.08 & 1.51 & 1.27 & 1.88 & 1.58 \\
\hline & 110 & 1.68 & 2.41 & 1.92 & 2.77 & 2.35 \\
\hline & 120 & 2.25 & 2.98 & 2.49 & 3.37 & 2.93 \\
\hline & 130 & 2.72 & 3.35 & 2.89 & 3.59 & 3.24 \\
\hline & 140 & 3.08 & 3.55 & 3.19 & 3.66 & 3.43 \\
\hline \multirow[t]{6}{*}{100} & 100 & 2.12 & 3.33 & 2.62 & 3.98 & 3.30 \\
\hline & 110 & 3.37 & 5.07 & 4.26 & 6.36 & 5.31 \\
\hline & 120 & 4.59 & 6.49 & 5.51 & 7.58 & 6.54 \\
\hline & 130 & 5.62 & 7.32 & 6.36 & 7.95 & 7.15 \\
\hline & 140 & 6.50 & 7.74 & 6.91 & 8.10 & 7.50 \\
\hline & 150 & 6.99 & 7.92 & 7.23 & 8.12 & 7.67 \\
\hline \multirow[t]{6}{*}{110} & 110 & 5.48 & 10 & 10 & 13.66 & 11.83 \\
\hline & 120 & 7.57 & 12.32 & 10.37 & 14.50 & 12.44 \\
\hline & 130 & 9.40 & 13.45 & 11.58 & 14.53 & 13.06 \\
\hline & 140 & 10.87 & 13.98 & 12.41 & 14.44 & 13.43 \\
\hline & 150 & 11.97 & 14.13 & 12.94 & 14.43 & 13.69 \\
\hline & 160 & 12.73 & 14.25 & 13.37 & 14.39 & 13.88 \\
\hline
\end{tabular}

${ }^{*}$ This table reports prices and bounds for an American min-call for various values of the underlying asset prices. Parameters values are $r=6 \%, \delta_{1}=\delta_{2}=5 \%, \rho=0, \sigma_{1}=\sigma_{2}=0.20, S_{2}=K=100$, $T=1$. LBA and UBA are estimated using Monte Carlo simulation with 50,000 paths and 100 discretization points. The optimization in LBA is performed using a grid search. The upper bound reported is obtained by substituting $H^{e}$ for $B^{m}$ and $B^{d}$ in the EEP representation. LUBA is the average of UBA and LBA (weight $\lambda=0.5$ ). 
bound approximation (UBA) and a lower-upper bound approximation (LUBA). Here LBA is computed by optimizing over the set of barriers $\left(L_{1}, L_{2}\right) \in$ $\{(102,102),(104,104), \ldots,(160,160)\}$. To calculate UBA we derive a threepoint lower bound $H^{e}=\left(H_{0}^{e}, H_{1 / 2}^{e}, H_{1}^{e}\right)$ using the method described in (9). This computation uses Monte Carlo simulation with 50,000 paths. LUBA is the average of UBA and LBA (with weight $\lambda=0.5$ ).

Note first that the lower bound approximation improves significantly upon the European min-call price. As long as $S^{1}$ is not too large it can also be verified that the upper bound improves significantly upon the value of an American call minoption on asset 2 alone. For instance, when $S^{1}=110, S^{2}=100$ the UBA improves by about $21 \%$ over the bound given by the call min-option on asset 2 . Note also that the binomial value computed with 100 time steps always lies in between the two bounds. Finally, the simple average of the two bounds (LUBA) is within a few percentage points of the binomial value.

4. Conclusion. In this paper we have examined the valuation of call options on the minimum of two dividend-paying assets. Simple stochastic dominance arguments enabled us to identify the geometric structure of the exercise region. It was shown that the optimal exercise boundary consists of three components, two surfaces in the region off the diagonal and one time-dependent curve along the diagonal. The structure of the exercise region and results from Rutkowski (1994) enabled us to identify the components of the min-option exercise premium. The most unusual aspect of this premium is perhaps the fact that the benefits from early exercise include a local time component related to the nonsmoothness of the payoff function. This is in sharp contrast with standard cases in which the exercise premium is entirely made up of dividends net of interest payments.

The early exercise premium representation also provides a simple way to characterize the exercise boundary. In that regard we showed that the components of the boundary satisfy a system of integral equations. In principle these can be used in a numerical scheme to compute the boundary and the option value. Alternatively, when computation time is an issue, one can rely on bounds derived from approximations of the optimal exercise policy. A lower bound for the option price was obtained based on a family of simple stopping times. This lower bound on the price was then used to construct a lower bound for the exercise boundary, which led to an upper bound for the min-option value. These price bounds provide an implementable alternative to the integral equation method suggested by the early exercise premium representation.

\section{APPENDIX: PROOFS}

Proof of Proposition 1. For (i) see Broadie and Detemple [(1997), Proposition A.5 (ray connectedness)]. The proof of (ii) follows from the inequality

$$
(x \wedge \lambda y-K)^{+} \leq \lambda(x \wedge y-K)^{+}+K(\lambda-1) \quad \text { for all } 1 \leq \lambda \leq x / y .
$$


See proofs in Broadie and Detemple [(1999), Proposition 1.4.1(2)] and Broadie and Detemple [(1997), Proposition 2.3]. Property (iii) holds since

$$
(y-K)^{+} \leq(\lambda x \wedge y-K)^{+} \leq(x \wedge y-K)^{+} \quad \text { for all } y / x \leq \lambda \leq 1 .
$$

Property (iv) follows from the fact that the min-option is less valuable than a standard call option written on $S^{2}$.

To prove (v) note that $(x \wedge y-K)^{+} \leq(w x+(1-w) y-K)^{+}$for all $w \in(0,1)$. The upper bound $C_{t}^{m}(x, y) \leq C_{t}^{w}(x, y)$ for the min-option price follows. Suppose that $(x, y) \in \mathcal{E}_{t}^{w}$ and $x=y$. Then $C_{t}^{m}(x, y) \leq(w x+(1-w) y-K)^{+}=(x-K)^{+}$. Since immediate exercise is feasible for the min-option, we get the lower bound $C_{t}^{m}(x, y) \geq(x \wedge y-K)^{+}=(x-K)^{+}$. We conclude that $C_{t}^{m}(x, y)=(x-K)^{+}$, that is, $(x, y) \in \varepsilon_{t}^{1}$.

Property (vi) follows from the properties of the payoff function described above and the characterization of the price function $C_{t}^{m}\left(S_{t}^{1}, S_{t}^{2}\right)$ in terms of the Snell envelope.

ProOf of Proposition 2. (i) This follows directly from Proposition 1(vi).

(ii) Let $B_{t}^{l}\left(x_{i}\right)=x_{i}, i=1,2$, where $x_{1}<x_{2}$. Let $x \in\left(x_{1}, x_{2}\right)$. Ray connectedness [Proposition 1(i)] implies that immediate exercise is optimal at $x$ if it is optimal at $x_{1}$. Assume that $B_{t}^{l}(x)<x$. Then ray connectedness implies $\left(\lambda x, \lambda B_{t}^{l}(x)\right) \in$ $\varepsilon_{t}^{1}$ for $\lambda=x_{2} / x$, that is, $\lambda B_{t}^{l}(x) \geq B_{t}^{l}\left(x_{2}\right)$. However, since $B_{t}^{l}(x)<x$, multiplying each side by $\lambda=x_{2} / x$ gives $\lambda B_{t}^{l}(x)<x_{2}=B_{t}^{l}\left(x_{2}\right)$. This contradicts the previous inequality. We conclude that $B_{t}^{l}(x)=x$.

(iii) This follows directly from Proposition $1(\mathrm{v})$.

(iv) Suppose that $x<y$. Then ray connectedness implies that $B_{t}^{l}(y) \leq \frac{y}{x} B_{t}^{l}(x)$. Hence $B_{t}^{l}(y)-B_{t}^{l}(x) \leq \frac{y-x}{x} B_{t}^{l}(x) \leq y-x$. Then $\left|B_{t}^{l}(y)-B_{t}^{l}(x)\right| \leq|y-x|$ and $B_{t}^{l}(x)$ is absolutely continuous. Hence $B_{t}^{l}(x)$ is differentiable almost everywhere.

(v) By proposition 1 (iv), $\lim _{t \rightarrow T} B_{t}^{l}(x) \leq \lim _{t \rightarrow T} B_{2}(t)$. Since $\lim _{t \rightarrow T} B_{2}(t)=$ $K \vee r K / \delta_{2}$ by (4), $\lim _{t \rightarrow T} B_{t}^{l}(x) \leq K \vee r K / \delta_{2}$. We prove that $B_{t}^{l}(x) \geq K \vee r K / \delta_{2}$ for all $t \in[0, T)$ and at all points $x$ such that $B_{t}^{l}(x)<x$. For every $t \in[0, T)$ and $\left(S_{t}^{1}, S_{t}^{2}\right) \in \mathcal{E}_{t}^{1}$ with $S_{t}^{1}>S_{t}^{2}$, it suffices to prove that $S_{t}^{2} \geq r K / \delta_{2}$.

Suppose not; that is, suppose that $\left(S_{t}^{1}, S_{t}^{2}\right) \in \mathcal{E}_{t}^{1}$, while $S_{t}^{1}>S_{t}^{2}$ and $S_{t}^{2}<$ $r K / \delta_{2}$. Since $C_{t}^{m}\left(S_{t}^{1}, S_{t}^{2}\right)>0$ it must be that $S_{t}^{2} \geq K$. Consider the stopping times

$$
\begin{aligned}
\tau_{1} & =\inf \left\{u \in[t, T): S_{u}^{2} \geq S_{u}^{1}\right\} \wedge T, \\
\tau_{2} & =\inf \left\{u \in[t, T): S_{u}^{2}=r K / \delta_{2}\right\} \wedge T
\end{aligned}
$$

and set $\tau=\tau_{1} \wedge \tau_{2}$. Then $\tau>t$ since $S_{t}^{1}>S_{t}^{2}$ and $S_{t}^{2}<r K / \delta_{2}$. Consider a portfolio consisting of 1 call min-option, 1 share of the stock $S^{2}$ held short and $K$ dollars invested at the risk-free rate. Suppose that we liquidate this portfolio at $\tau$ (the option is exercised at liquidation). The cash flows generated by this 
TABLE 2

\begin{tabular}{lcc}
\hline & Time $\boldsymbol{t}$ & Time $\boldsymbol{\tau}=\boldsymbol{\tau}_{\mathbf{1}} \wedge \boldsymbol{\tau}_{\mathbf{2}}$ \\
\hline Buy call & $-\left(S_{t}^{2}-K\right)$ & $S_{\tau}^{2}-K$ \\
Sell stock & $+S_{t}^{2}$ & $-S_{\tau}^{2}-\int_{t}^{\tau} e^{r(\tau-v)} \delta_{2} S_{v}^{2} d v$ \\
Invest $K$ & $-K$ & $K e^{r(\tau-t)}=K+\int_{t}^{\tau} e^{r(\tau-v)} r K d v$ \\
Total & 0 & $-\int_{t}^{\tau} e^{r(\tau-v)}\left(\delta_{2} S_{v}^{2}-r K\right) d v$ \\
\hline
\end{tabular}

policy are shown in Table 2. Since $r K-\delta_{2} S_{v}^{2}>0$ for all $v \in[t, \tau)$ this policy is an arbitrage opportunity. Thus, we must have $S_{t}^{2} \geq r K / \delta_{2}$ when $\left(S_{t}^{1}, S_{t}^{2}\right) \in \varepsilon_{t}^{1}$. A similar argument shows that $\lim _{t \rightarrow T} B_{t}^{u}=K \vee r K / \delta_{1}$.

To show that $\lim _{t \rightarrow T} B_{t}^{e}=K$, we use the fact that $C_{t}^{m}(x, y)$ is a nonincreasing function of dividends. Let $\delta=\left(\delta_{1}, \delta_{2}\right)$ and write $C_{t}^{m}(x, y ; \delta)$ to indicate the dependence on dividends. Similarly, let $\mathcal{E}(\delta), \mathcal{E}_{t}(\delta), \mathcal{E}_{t}^{1}(\delta), \mathcal{E}_{t}^{2}(\delta)$ be the corresponding subsets of the exercise region. Then $C_{t}^{m}(x, y ; \delta) \leq C_{t}^{m}\left(x, y ; \mathbf{0}_{2}\right)$ for all $(x, y) \in \mathbb{R}^{+} \times \mathbb{R}^{+}$, where $\mathbf{0}_{2}=(0,0)$.

Consider a point $\left(S_{t}, S_{t}\right)$ on the diagonal and suppose that immediate exercise is optimal for the model without dividends, that is, $\left(S_{t}, S_{t}\right) \in \mathcal{E}_{t}\left(\mathbf{0}_{2}\right)=$ $\varepsilon_{t}^{1}\left(\mathbf{0}_{2}\right) \cap \varepsilon_{t}^{2}\left(\mathbf{0}_{2}\right)$ [see Villeneuve (1999)]. Then $S_{t} \wedge S_{t}-K \leq C_{t}^{m}\left(S_{t}, S_{t} ; \delta\right) \leq$ $C_{t}^{m}\left(S_{t}, S_{t} ; \mathbf{0}_{2}\right)=S_{t} \wedge S_{t}-K$ and therefore $\left(S_{t}, S_{t}\right) \in \mathcal{E}_{t}(\delta)$. We conclude that $\varepsilon_{t}^{1}(\delta) \cap \varepsilon_{t}^{2}(\delta) \supseteq \varepsilon_{t}^{1}\left(\mathbf{0}_{2}\right) \cap \varepsilon_{t}^{2}\left(\mathbf{0}_{2}\right)$. Thus $K \leq B_{t}^{e}(\delta) \leq B_{t}^{e}\left(\mathbf{0}_{2}\right)$. The result now follows from Proposition 2.4 in Villeneuve (1999), which shows that $B_{t}^{e}\left(\mathbf{0}_{2}\right) \rightarrow K$ as $t \rightarrow T$.

(vi) By property (iv) we have $\left|B_{t}^{l}(y)-B_{t}^{l}(x)\right| \leq|y-x|$. Thus, with $B^{l}(t, \cdot) \equiv$ $B_{t}^{l}(\cdot)$,

$$
\begin{aligned}
\left|B^{l}(t, y)-B^{l}(s, x)\right| & \leq\left|B^{l}(t, y)-B^{l}(t, x)\right|+\left|B^{l}(t, x)-B^{l}(s, x)\right| \\
& \leq|y-x|+\left|B^{l}(t, x)-B^{l}(s, x)\right|
\end{aligned}
$$

and it suffices to show that $B^{l}(\cdot, x)$ is a continuous function for any fixed $x$.

By the continuity of the value function and the definition of $B^{l}$, it follows that

$$
\lim _{u \downarrow t} B^{l}(u, x)-K=\lim _{u \downarrow t} C_{u}^{m}\left(x, B^{l}(u, x)\right)=C_{t}^{m}\left(x, \lim _{u \downarrow t} B^{l}(u, x)\right) \geq B_{t}^{l}(x)-K
$$

(the inequality on the right-hand side follows from the fact that the exercise region is a closed set). Thus, $\sup _{u>t} B_{u}^{l}(x) \geq B_{t}^{l}(x)$ and, since $B_{v}^{l}(x) \leq B_{t}^{l}(x)$ for $v \geq t$, we conclude that $B^{l}(\cdot, x)$ is right-continuous.

To prove left-continuity, we define $\eta(x)=\inf _{u<t} B^{l}(u, x)$, and want to show that $\eta(x)=B^{l}(t, x)$. First, for any $u \in[0, t]$,

$$
B^{l}(u, x)-K=C_{u}^{m}\left(x, B^{l}(u, x)\right) \geq C_{t}^{m}\left(x, B^{l}(u, x)\right)
$$


by Proposition 1 (vi). So $C_{t}^{m}\left(x, B^{l}(u, x)\right)=B^{l}(u, x)-K$ by a no-arbitrage argument. Then $B^{l}(u, x) \geq B^{l}(t, x)$ and thus $\eta(x) \geq B^{l}(t, x)$. Second,

$$
\inf _{y>x} \eta(y)=\inf _{y>x} \inf _{u<t} B^{l}(u, y)=\inf _{u<t} \inf _{y>x} B^{l}(u, y)=\inf _{u<t} B^{l}(u, x)=\eta(x)
$$

because $B^{l}(u, x)$ is nondecreasing and continuous for each $u$ by Proposition 2(i) and (iv). Then $\eta(x)$ is right-continuous. If the set $W=\left\{x: \eta(x) \neq B^{l}(t, x)\right\} \neq \varnothing$, then there is an open set contained in the boundary curve $y=B^{l}(t, x)$ and over which $\eta(x)>B^{l}(t, x)$ since $B^{l}(t, \cdot)$ is continuous and bounded by $\eta(x)$. Therefore there is an open set $V \subseteq \mathbb{R}^{+} \times \mathbb{R}^{+}$such that for every $(x, y) \in V$ we have $B^{l}(t, x)<y<\eta(x)$.

Let us now apply the usual variational approach to price the option. Write $£$ as the Black-Scholes/Merton differential operator (see Proposition 9 below). By Jaillet, Lamberton and Lapeyre [(1990), Theorems 3.1 and 3.2], we have that $£ C^{m} \leq 0$ in the sense of distribution in the open set $[0, T) \times\left(\mathbb{R}^{+}\right)^{2}$, and $£ C^{m}=0$ in the sense of distribution in the continuation region $\bigcup_{t \in[0, T)} \mathcal{C}_{t}$, where $\mathcal{C}_{t}=\varepsilon_{t}^{c}$.

For $y<\eta(x)$, we have $\eta(x)=\inf _{u<t} B^{l}(u, x) \leq \lim _{n \rightarrow \infty} B^{l}\left(u_{n}, x\right)$ for all sequences $u_{n} \uparrow t$, and $(x, y) \in \mathcal{C}_{u_{n}}$. Since $£ C^{m}=0$ for $(x, y) \in \mathcal{C}_{u_{n}}$ in distribution, by the bounded convergence theorem and the continuity of $C^{m}$ we see that $£ C^{m}=0$ over $(x, y) \in \mathcal{C}_{t}$ in distribution. In particular, $£ C^{m}=0$ over $V$ in distribution.

Let $£_{1}$ denote the sum of the second order terms in $£$. In $\mathcal{C}$ we have

$$
£_{1} C^{m}=r C^{m}-\left(r-\delta_{1}\right) x \frac{\partial C^{m}}{\partial x}-\left(r-\delta_{2}\right) y \frac{\partial C^{m}}{\partial y}-\frac{\partial C^{m}}{\partial t} .
$$

Recall that $\partial C^{m} / \partial t<0$ and that $C^{m} \rightarrow S_{1} \wedge S_{2}-K, \min \left(C_{x}^{m}, C_{y}^{m}\right) \rightarrow 0$, $\max \left(C_{x}^{m}, C_{y}^{m}\right) \rightarrow 1$ when approaching the boundary. Also recall that $\delta_{1} x-r K>0$ in $\mathscr{E} \cap\{x<y\}$ and $\delta_{2} y-r K>0$ in $\mathscr{E} \cap\{y<x\}$. Combining all these elements shows that $£_{1} C^{m}>0$ when approaching the boundary from within $C$.

For any $t, x$ and $\delta>0, \varepsilon>0$, let

$A_{\delta, \varepsilon}=\left\{(s, u, y): t-\varepsilon<s<t, x-\delta<u<x+\delta,\left(B_{s}^{l}(u)-\varepsilon\right) \vee K<y<B_{s}^{l}(u)\right\}$.

Define $\mathcal{C}_{\delta, \varepsilon}$ to be the closure of the intersection of $\mathcal{C}$ and $A_{\delta, \varepsilon}$. Let $\mathcal{C}_{s, \delta, \varepsilon}=$ $\left\{(u, y):(s, u, y) \in \mathcal{C}_{\delta, \varepsilon}\right\}$. From the continuity of $£_{1}$ over $\mathcal{C}_{\delta, \varepsilon}$, we get that $£_{1} C^{m}$ is uniformly positive over $\mathcal{C}_{\delta, \varepsilon}$ by choosing $\delta$ and $\varepsilon$ small enough. Since, for any $t-\varepsilon<s<t$, we have $C^{m}\left(s, x, B_{s}^{l}(x)\right)=B_{s}^{l}(x)-K$ and since $B_{s}^{l}(x)$ is continuous in $x$, we can choose $\delta$ small enough such that $C^{m}(s, u, y)=C^{m}(s, x, y)$ for all $|u-x|<\delta$. Hence the terms in $£_{1}$ involving derivatives with respect to $x$ vanish over $\mathcal{C}_{s, \delta, \varepsilon}$.

Finally, for any $s \in(t-\varepsilon, t)$ and $\left(B_{s}^{l}(x)-\varepsilon\right) \vee K<y<B_{s}^{l}(x)$, we have

$$
\begin{aligned}
C^{m}(s, x, y)-(x \wedge y-K)^{+} & =\int_{y}^{B_{s}^{l}(x)} \int_{z}^{B_{s}^{l}(x)} \frac{\partial^{2} C^{m}(s, x, v)}{\partial v^{2}} d v d z \\
& \geq \frac{\varepsilon^{2}}{\sigma_{2}^{2} B_{s}^{l}(x)} .
\end{aligned}
$$


Letting $s$ approach $t$ from the left, we get that $\left(t, x, B_{t-}^{l}(x)-\varepsilon\right) \in \mathcal{C}$ for all $\varepsilon$. Hence $B_{t-}^{l}(x) \leq B_{t}^{l}(x)$ and thus the left-continuity since $B_{t}^{l}(x) \equiv B^{l}(t, x)$ is a nonincreasing function of time.

Combining the results above we conclude that $B^{l}(t, x)$ is continuous. This completes the proof of Proposition 2.

Proof of Theorem 3. Consider the function $g(x, y)=x \wedge y$ on $\mathbb{R}^{2}$. For any $n \geq 1$, set

$$
g_{n}(x, y)= \begin{cases}x, & x \leq y-\frac{1}{n} \\ x-\frac{n}{4}\left(x-y+\frac{1}{n}\right)^{2}, & y-\frac{1}{n}<x<y, \\ y-\frac{n}{4}\left(y-x+\frac{1}{n}\right)^{2}, & y \leq x<y+\frac{1}{n} \\ y, & x \geq y+\frac{1}{n} .\end{cases}
$$

Then, for any $x, y$, we have $\lim _{n \rightarrow \infty} g_{n}(x, y)=g(x, y)$.

By direct calculation, we get

$$
\begin{aligned}
\frac{\partial g_{n}}{\partial x}(x, y)= \begin{cases}1, & x \leq y-\frac{1}{n}, \\
1-\frac{n}{2}\left(x-y+\frac{1}{n}\right), & y-\frac{1}{n}<x<y, \\
\frac{n}{2}\left(y-x+\frac{1}{n}\right), & y \leq x<y+\frac{1}{n}, \\
0, & x \geq y+\frac{1}{n},\end{cases} \\
\frac{\partial g_{n}}{\partial y}(x, y)= \begin{cases}0, & x \leq y-\frac{1}{n}, \\
\frac{n}{2}\left(x-y+\frac{1}{n}\right), & y \leq x<y \\
1-\frac{n}{2}\left(y-x+\frac{1}{n}\right), & x \geq y+\frac{1}{n}, \\
1, & \quad x \leq y-\frac{1}{n},\end{cases} \\
\frac{\partial^{2} g_{n}}{\partial x \partial y}(x, y)= \begin{cases}0, & y-\frac{1}{n}<x<y, \\
\frac{n}{2}, & y \leq x<y+\frac{1}{n},\end{cases}
\end{aligned}
$$


and

$$
\frac{\partial^{2} g_{n}}{\partial x^{2}}(x, y)=\frac{\partial^{2} g_{n}}{\partial y^{2}}(x, y)=-\frac{\partial^{2} g_{n}}{\partial x \partial y}(x, y)= \begin{cases}0, & |x-y| \geq \frac{1}{n}, \\ -\frac{n}{2}, & |x-y|<\frac{1}{n} .\end{cases}
$$

Hence $g_{n}$ has continuous partial derivatives of the first order, and the second order derivatives are continuous for $|x-y| \neq 1 / n$.

Let

$$
h(x, y)= \begin{cases}C \exp \left[\frac{1}{\left(x^{2}+y^{2}-1\right)}\right], & \sqrt{x^{2}+y^{2}}<1, \\ 0, & \sqrt{x^{2}+y^{2}} \geq 1,\end{cases}
$$

where $C=\left(\int_{\sqrt{x^{2}+y^{2}}<1} \exp \left[1 /\left(x^{2}+y^{2}-1\right)\right] d x d y\right)^{-1}$. Clearly $h$ is a $C^{\infty}$ function supported on the closed unit ball. For any $m \geq 1$, define

$$
\begin{aligned}
& h_{m}(x, y)=m^{2} h(m x, m y), \\
& g_{n}^{m}(x, y)=\int_{R^{2}} g_{n}\left(x-x_{1}, y-y_{1}\right) h_{m}\left(x_{1}, y_{1}\right) d x_{1} d y_{1} .
\end{aligned}
$$

Our first lemma shows that $g_{n}^{m}$ is a smooth approximation of $g_{n}$.

LEMMA 7. The function $g_{n}^{m}$ is a $C^{\infty}$ function, and for every $n$ the functions $g_{n}^{m}, \frac{\partial g_{n}^{m}}{\partial x}, \frac{\partial g_{n}^{m}}{\partial y}$ converge uniformly to $g_{n}, \frac{\partial g_{n}}{\partial x}, \frac{\partial g_{n}}{\partial y}$, respectively, as $m$ goes to infinity. For $|x-y| \neq 1 / n$, we have

(10) $\lim _{m \rightarrow \infty} \frac{\partial^{2} g_{n}^{m}}{\partial x^{2}}=\frac{\partial^{2} g_{n}}{\partial x^{2}}, \quad \lim _{m \rightarrow \infty} \frac{\partial^{2} g_{n}^{m}}{\partial y^{2}}=\frac{\partial^{2} g_{n}}{\partial y^{2}}, \quad \lim _{m \rightarrow \infty} \frac{\partial^{2} g_{n}^{m}}{\partial x \partial y}=\frac{\partial^{2} g_{n}}{\partial x \partial y}$.

Proof. First note that

$$
g_{n}^{m}(x, y)=\int_{R^{2}} g_{n}\left(x_{1}, y_{1}\right) h_{m}\left(x-x_{1}, y-y_{1}\right) d x_{1} d y_{1} .
$$

This combined with the $C^{\infty}$ property of $h_{m}$ implies that $g_{n}^{m}$ is a $C^{\infty}$ function. The limits in (10) follow from the fact that the integral and differential operations are interchangeable.

By direct calculation, one gets that

$$
\begin{aligned}
& \left|g_{n}^{m}(x, y)-g_{n}(x, y)\right| \\
& \quad=\left|\int_{R^{2}}\left[g_{n}\left(x-x_{1}, y-y_{1}\right)-g_{n}(x, y)\right] h_{m}\left(x_{1}, y_{1}\right) d x_{1} d y_{1}\right| \\
& \quad \leq\left|\int_{R^{2}}\left[g_{n}\left(x-x_{1}, y-y_{1}\right)-g_{n}\left(x-x_{1}, y\right)\right] h_{m}\left(x_{1}, y_{1}\right) d x_{1} d y_{1}\right|
\end{aligned}
$$




$$
\begin{aligned}
& \quad+\left|\int_{R^{2}}\left[g_{n}\left(x-x_{1}, y\right)-g_{n}(x, y)\right] h_{m}\left(x_{1}, y_{1}\right) d x_{1} d y_{1}\right| \\
& \leq \frac{1}{m}+\frac{1}{m}
\end{aligned}
$$

where in the last inequality we used the fact that the partial derivatives of $g_{n}$ are bounded by 1 . Thus $g_{n}^{m}$ converges to $g_{n}$ uniformly as $m$ goes to infinity. The uniform convergence of $\left(\frac{\partial g_{n}^{m}}{\partial x}, \frac{\partial g_{n}^{m}}{\partial y}\right)$ to $\left(\frac{\partial g_{n}}{\partial x}, \frac{\partial g_{n}}{\partial y}\right)$ can be proved in the same manner. The only difference is that the one-sided derivatives are used when the second order derivatives do not exist.

Now consider the Itô processes $d x_{t}=\left(r-\delta_{1}-\frac{1}{2} \sigma_{1}^{2}\right) d t+\sigma_{1} d z_{t}^{1}$ and $d y_{t}=$ $\left(r-\delta_{2}-\frac{1}{2} \sigma_{2}^{2}\right) d t+\sigma_{2} d z_{t}^{2}$. Our next lemma provides the decomposition of the process $x \wedge y$.

LEMMA 8. The process $x_{t} \wedge y_{t}$ is a semimartingale with the following decomposition:

$$
\begin{aligned}
x_{t} \wedge y_{t}-x_{0} \wedge y_{0}= & \int_{0}^{t}\left(r-\delta_{1}-\frac{1}{2} \sigma_{1}^{2}\right) \chi_{\left\{x_{u}<y_{u}\right\}} d u \\
& +\int_{0}^{t}\left(r-\delta_{2}-\frac{1}{2} \sigma_{2}^{2}\right) \chi_{\left\{x_{u}>y_{u}\right\}} d u \\
& -\frac{1}{2}\left(\sigma_{1}^{2}-2 \sigma_{1} \rho \sigma_{2}+\sigma_{2}^{2}\right) L(t, 0 ; x-y) \\
& +\int_{0}^{t} \sigma_{1} \chi_{\left\{x_{u}<y_{u}\right\}} d z_{u}^{1}+\int_{0}^{t} \sigma_{2} \chi_{\left\{x_{u}>y_{u}\right\}} d z_{u}^{2},
\end{aligned}
$$

where $L(t, 0 ; x-y)$ is the local time of the semimartingale $x_{t}-y_{t}$ at 0 .

PROOF. By Itô's formula, we have

$$
g_{n}^{m}\left(x_{t}, y_{t}\right)=g_{n}^{m}\left(x_{0}, y_{0}\right)+I\left(g_{n}^{m}\right)+I I\left(g_{n}^{m}\right)+I I I\left(g_{n}^{m}\right),
$$

where, for a function $g$,

$$
\begin{aligned}
I(g) & =\int_{0}^{t} \frac{\partial g}{\partial x}\left(r-\delta_{1}-\frac{1}{2} \sigma_{1}^{2}\right) d u+\int_{0}^{t} \frac{\partial g}{\partial y}\left(r-\delta_{2}-\frac{1}{2} \sigma_{2}^{2}\right) d u \\
I I(g) & =\int_{0}^{t} \frac{\partial g}{\partial x} \sigma_{1} d z_{u}^{1}+\int_{0}^{t} \frac{\partial g}{\partial y} \sigma_{2} d z_{u}^{2} \\
I I I(g) & =\frac{1}{2} \int_{0}^{t} \frac{\partial^{2} g}{\partial x^{2}} \sigma_{1}^{2} d u+\int_{0}^{t} \frac{\partial^{2} g}{\partial x \partial y} \sigma_{1} \rho \sigma_{2} d u+\frac{1}{2} \int_{0}^{t} \frac{\partial^{2} g}{\partial y^{2}}\left(\sigma_{2}\right)^{2} d u
\end{aligned}
$$

By letting $m$ go to infinity, we clearly have that $g_{n}^{m}\left(x_{t}, y_{t}\right)$ converges almost surely to $g_{n}\left(x_{t}, y_{t}\right)$ for each $t$. By the dominated convergence theorem we have, 
almost surely, that

$$
\lim _{m \rightarrow \infty} I\left(g_{n}^{m}\right)=I\left(g_{n}\right), \quad \lim _{m \rightarrow \infty} I I I\left(g_{n}^{m}\right)=I I I\left(g_{n}\right) .
$$

By the isometry and the dominated convergence theorem, one gets that $I I\left(g_{n}^{m}\right)$ converges to $I I\left(g_{n}\right)$ in $L^{2}$, which leads to the almost sure convergence of a subsequence. Hence the following holds almost surely:

$$
g_{n}\left(x_{t}, y_{t}\right)=g_{n}\left(x_{0}, y_{0}\right)+I\left(g_{n}\right)+I I\left(g_{n}\right)+I I I\left(g_{n}\right) .
$$

By direct calculation, we get

$$
\begin{gathered}
\left|g\left(x_{t}, y_{t}\right)-g_{n}\left(x_{t}, y_{t}\right)\right| \leq \frac{1}{n}, \\
E \int_{0}^{t}\left[\left|\frac{\partial g_{n}}{\partial x}-\chi_{\{x<y\}}\right|+\left|\frac{\partial g_{n}}{\partial y}-\chi_{\{x>y\}}\right|\right]^{2} d u \leq \int_{0}^{t} P\left\{\left|x_{u}-y_{u}\right| \leq \frac{1}{n}\right\} d u \rightarrow 0,
\end{gathered}
$$

which implies

$$
\begin{gathered}
g_{n}\left(x_{t}, y_{t}\right)-g_{n}\left(x_{0}, y_{0}\right) \rightarrow g\left(x_{t}, y_{t}\right)-g\left(x_{0}, y_{0}\right) \quad \text { in } L^{2}, \\
I\left(g_{n}\right) \rightarrow \int_{0}^{t}\left(r-\delta_{1}-\frac{1}{2} \sigma_{1}^{2}\right) \chi_{\left\{x_{u}<y_{u}\right\}} d u+\int_{0}^{t}\left(r-\delta_{2}-\frac{1}{2} \sigma_{2}^{2}\right) \chi_{\left\{x_{u}>y_{u}\right\}} d u \quad \text { in } L^{2}, \\
I I\left(g_{n}\right) \rightarrow \int_{0}^{t} \sigma_{1} \chi_{\left\{x_{u}<y_{u}\right\}} d z_{u}^{1}+\int_{0}^{t} \sigma_{2} \chi_{\left\{x_{u}>y_{u}\right\}} d z_{u}^{2} \quad \text { in } L^{2} .
\end{gathered}
$$

For $I I I\left(g_{n}\right)$, we have

$$
\begin{aligned}
I I I\left(g_{n}\right) & =-\frac{n}{4} \int_{0}^{t}\left(\sigma_{1}^{2}-2 \sigma_{1} \rho \sigma_{2}+\sigma_{2}^{2}\right) \chi\left\{\left|x_{u}-y_{u}\right|<1 / n\right\} d u \\
& \rightarrow-\frac{1}{2}\left(\sigma_{1}^{2}-2 \sigma_{1} \rho \sigma_{2}+\sigma_{2}^{2}\right) L(t, 0 ; x-y),
\end{aligned}
$$

where

$$
L(t, 0 ; x-y)=\lim _{n \rightarrow \infty} n / 2 \int_{0}^{t} \chi_{\left\{\left|x_{u}-y_{u}\right|<1 / n\right\}} d u
$$

is the local time of the semimartingale $x_{t}-y_{t}$ at 0 measured in $t$, and the limit is in $L^{2}$. This completes the proof of the lemma.

We now complete the proof of the theorem. Recall that $S_{t}^{1}=e^{x_{t}}, S_{t}^{2}=e^{y_{t}}$. If we set $X_{t}=S_{t}^{1} \wedge S_{t}^{2}$ it is clear that $X_{t}=e^{x_{t} \wedge y_{t}}$. By applying Itô's formula again, it follows that

$$
\begin{aligned}
X_{t}-X_{0}= & \int_{0}^{t} S_{u}^{1}\left(r-\delta_{1}\right) \chi_{\left\{x_{u}<y_{u}\right\}} d u+\int_{0}^{t} S_{u}^{2}\left(r-\delta_{2}\right) \chi_{\left\{x_{u}>y_{u}\right\}} d u \\
& -\frac{1}{2}\left(\sigma_{1}^{2}-2 \sigma_{1} \rho \sigma_{2}+\sigma_{2}^{2}\right) \int_{0}^{t} X_{u} d L(u, 0 ; x-y) \\
& +\int_{0}^{t} \sigma_{1} S_{u}^{1} \chi_{\left\{x_{u}<y_{u}\right\}} d z_{u}^{1}+\int_{0}^{t} \sigma_{2} S_{u}^{2} \chi_{\left\{x_{u}>y_{u}\right\}} d z_{u}^{2} .
\end{aligned}
$$


For any fixed $t$, let $F(s, t, X)=e^{-r(s-t)}(X-K)^{+}, t \leq s \leq T$. Since $F$ is convex in $X$ and $X_{S}$ is a one-dimensional continuous semimartingale, we can apply Theorem 3.7.1 in Karatzas and Shreve (1987) and get

$$
\begin{aligned}
F\left(s, t, X_{s}\right)= & F\left(t, t, X_{t}\right)+\int_{t}^{s}(-r) F\left(u, t, X_{u}\right) d u+\int_{t}^{s}(r) F\left(u, t, X_{u}\right) d u \\
& +\int_{t}^{s} \chi_{\left\{X_{u}>K\right\}} e^{-r(u-t)} r K d u+\frac{1}{2} \int_{t}^{s} e^{-r(u-t)} d L(u, 0 ; X-K) \\
& -\int_{t}^{s} \chi_{\left\{X_{u}>K\right\}} e^{-r(u-t)}\left[\delta_{1} S_{u}^{1} \chi_{\left\{S_{u}^{1}<S_{u}^{2}\right\}}+\delta_{2} S_{u}^{2} \chi_{\left\{S_{u}^{1}>S_{u}^{2}\right\}}\right] d u \\
& -\frac{1}{2}\left(\sigma_{1}^{2}-2 \sigma_{1} \rho \sigma_{2}+\sigma_{2}^{2}\right) \int_{t}^{s} \chi_{\left\{X_{u}>K\right\}} e^{-r(u-t)} X_{u} d L(u, 0 ; x-y) \\
& +\int_{t}^{s} \chi_{\left\{X_{u}>K\right\}} e^{-r(u-t)}\left[\sigma_{1} S_{u}^{1} \chi_{\left\{S_{u}^{1}<S_{u}^{2}\right\}} d z_{u}^{1}+\sigma_{2} S_{u}^{2} \chi_{\left\{S_{u}^{1}>S_{u}^{2}\right\}} d z_{u}^{2}\right],
\end{aligned}
$$

where $L(s, 0 ; X-K)$ is the local time of process $X-K$ at zero. This combined with Proposition A.1 in Rutkowski (1994) implies the result.

Our next proposition provides properties of the price function which will be useful in proving the uniqueness of the solution.

Proposition 9. (i) The price function $C_{t}^{m}\left(S_{t}^{1}, S_{t}^{2}\right)$ satisfies the boundedness conditions

$$
\begin{aligned}
& \left|C_{t}^{m}(x, y)-C_{t}^{m}\left(x^{\prime}, y\right)\right| \leq\left|x-x^{\prime}\right|, \\
& \left|C_{t}^{m}(x, y)-C_{t}^{m}\left(x, y^{\prime}\right)\right| \leq\left|y-y^{\prime}\right|
\end{aligned}
$$

and $\partial C^{m} / \partial t$ is locally bounded.

(ii) In the continuation region, $C^{m}$ has continuous second-order partial derivatives and $£ C^{m}=0$, where

$$
\begin{aligned}
£= & \left(r-\delta_{1}\right) x \frac{\partial}{\partial x}+\left(r-\delta_{2}\right) y \frac{\partial}{\partial y}-\frac{\partial}{\partial t}-r \\
& +\frac{1}{2}\left[\sigma_{1}^{2} x^{2} \frac{\partial^{2}}{\partial x^{2}}+2 \rho \sigma_{1} \sigma_{2} x y \frac{\partial^{2}}{\partial x \partial y}+\sigma_{2}^{2} y^{2} \frac{\partial^{2}}{\partial y^{2}}\right] .
\end{aligned}
$$

Proof. (i) The proof parallels Broadie and Detemple [(1997), Proposition 2.6] by using $\left|(x \wedge a-K)^{+}-(y \wedge a-K)^{+}\right| \leq|x-y|$.

(ii) Pick any point $P=\left(x_{0}, y_{0}, t_{0}\right) \in \mathcal{C}$, the continuation region. Choose an open rectangle $R=\left(x_{1}, x_{2}\right) \times\left(y_{1}, y_{2}\right) \times\left(t_{1}, t_{2}\right) \subseteq \mathcal{C}$ such that $P \in R$. By Friedman (1964) there exists a unique solution $\phi$ to the following Cauchy problem:

$$
\begin{array}{clrl}
£ \phi & =0 & \forall(x, y, t) & \in R, \\
\phi(x, y, T-t) & =C_{t}^{m}(x, y) & \forall(x, y, t) \in \partial R .
\end{array}
$$


We must show that $\phi=C^{m}$ in $R$; hence $£ C^{m}=0$ in $\mathcal{C}$. Let $N_{u}=e^{-r(u-t)} \phi\left(S_{u}^{1}\right.$, $\left.S_{u}^{2}, T-u\right)$ and consider the stopping times

$$
\begin{aligned}
\tau & =\inf \left\{u \geq t:\left(S_{u}^{1}, S_{u}^{2}, u\right) \notin R\right\}, \\
\tau^{\prime} & =\inf \left\{u \geq t:\left(S_{u}^{1}, S_{u}^{2}, u\right) \notin \mathcal{C}\right\} .
\end{aligned}
$$

Then $\tau \leq \tau^{\prime}$. By Itô's rule, the stopped process $N_{u \wedge \tau}$ is a bounded martingale. Hence

$$
\begin{aligned}
\phi\left(S_{t}^{1}, S_{t}^{2}, T-t\right) & =E_{t}^{*}\left[e^{-r(\tau-t)} \phi\left(S_{\tau}^{1}, S_{\tau}^{2}, T-\tau\right)\right] \\
& =E_{t}^{*}\left[e^{-r(\tau-t)} C_{\tau}^{m}\left(S_{\tau}^{1}, S_{\tau}^{2}\right)\right]=C_{t}^{m}\left(S_{t}^{1}, S_{t}^{2}\right),
\end{aligned}
$$

where the last step follows from the fact that $\tau \leq \tau^{\prime}$. This establishes $£ C^{m}=0$ in $\mathcal{C}$. The continuity of the second-order partials then follows from (i) and the continuity of the coefficients of the model.

This completes the proof of the proposition.

REMARK 2. For standard call options on a single underlying asset price, the local time integral vanishes because the exercise boundary lies above the strike $K$. The smoothness of both the price function and the exercise boundary have been shown by McKean (1965) and van Moerbeke (1976).

ProOf of THEOREM 4. To prove the theorem we first establish two auxiliary lemmas.

Consider a quadruplet of continuous functions $\left\{\phi(x, y, t), H_{t}^{l}(x), H_{t}^{u}(y), H_{t}^{e}\right\}$, where

$$
\begin{aligned}
H_{t}^{l}(x) & =\inf \{y: x \leq y, \phi(x, y, t)=g(x, y)\}, \\
H_{t}^{u}(y) & =\inf \{x: y \leq x, \phi(x, y, t)=g(x, y)\}, \\
H_{t}^{e} & =\inf \{x: x>0, \phi(x, x, t)=g(x, x)\},
\end{aligned}
$$

and where $g(x, y)=(x \wedge y-K)^{+}$. Define the associated exercise set $\varepsilon_{t}(H)$ by $(x, y, t) \in \varepsilon_{t}(H)$ if and only if $x=y \geq H_{t}^{e}$ or $H_{t}^{l}(x) \leq y<x$ or $H_{t}^{u}(y) \leq x<y$. The continuation set $\mathcal{C}_{t}(H)$ is defined as the complement of $\varepsilon_{t}(H)$. The following result holds.

LEMMA 10. Consider the system of equations (12) in four unknown functions $\left\{\phi(x, y, t), H_{t}^{l}(x), H_{t}^{u}(y), H_{t}^{e}\right\}$,

$$
\begin{array}{rlrl}
£ \phi & =0 & & \forall(x, y, t) \in \mathcal{C}(H), 0 \leq t<T, \\
\phi(x, y, 0) & =g(x, y), & & \\
\phi(x, y, t) & =g(x, y) & \forall(x, y, t) \in \mathcal{E}_{t}(H), \\
\phi(x, y, t) & >g(x, y) & \forall(x, y, t) \in \mathcal{C}_{t}(H) .
\end{array}
$$

Then $\left(C^{m}, B_{t}^{l}(\cdot), B_{t}^{u}(\cdot), B_{t}^{e}\right)$ is a solution of (12). Moreover, $C^{m}$ is the maximal 
element among all the solutions of (12); that is, if $\left(\overline{C_{t}^{m}}, \overline{B_{t}^{l}(\cdot)}, \overline{B_{t}^{u}(\cdot)}, \overline{B_{t}^{e}}\right)$ is another solution of (12), then $\overline{C^{m}} \leq C^{m}$.

ProOF. By the last proposition it is clear that $\left(C_{t}^{m}, B_{t}^{l}(\cdot), B_{t}^{u}(\cdot), B_{t}^{e}\right)$ is a solution of (12). Let $\left(\overline{C_{t}^{m}}, \overline{B_{t}^{l}(\cdot)}, \overline{B_{t}^{u}(\cdot)}, \overline{B_{t}^{e}}\right)$ denote another solution with the corresponding exercise region $\overline{\varepsilon_{t}}$. We prove that $\overline{C^{m}} \leq C^{m}$. In its continuation region $\overline{\mathcal{C}}$, applying the same proof as in Proposition 9(ii), $\overline{C_{t}^{m}}(x, y)=E_{t}^{*}\left[e^{-r(\tau-t)} g\left(S_{\tau}^{1}, S_{\tau}^{2}\right)\right]$ for some stopping time $\tau \in \delta_{t, T}$. Hence $\overline{C^{m}} \leq C^{m}$ by the optimality property of the price function $C^{m}$. In the common exercise region $\varepsilon \cap \bar{\varepsilon}, \overline{C^{m}}=C^{m}=g(x, y)$. If $(x, y, t) \in \overline{\mathcal{E}}-\mathcal{E}$, then $\overline{C^{m}}=g<C_{t}^{m}(x, y)$ by the last boundary condition of (12). This completes the proof.

Lemma 10 shows that the price function is maximal in a set of solutions. A refinement of this result is demonstrated next.

LEMMA 11. $\left(C_{t}^{m}, B_{t}^{l}(\cdot), B_{t}^{u}(\cdot), B_{t}^{e}\right)$ is the unique solution of the system of equations (12) subject to the following conditions:

$$
\begin{aligned}
& \lim _{t \rightarrow T} B_{t}^{l}(x)=K \vee r K / \delta_{2} \quad \text { for } B_{t}^{l}(x)<x ; \\
& \lim _{t \rightarrow T} B_{t}^{u}(y)=K \vee r K / \delta_{1} \quad \text { for } B_{t}^{u}(y)<y \text {; } \\
& \lim _{t \rightarrow T} B_{t}^{e}=K
\end{aligned}
$$

and $B_{t}^{l}(x), B_{t}^{u}(y)$ are continuous nonincreasing functions of time.

This lemma establishes the uniqueness of the solution of the two-dimensional free boundary problem. The proof is based on martingale arguments.

ProOf OF Lemma 11. Suppose we have another solution $\left(\overline{C_{t}^{m}}, \overline{B_{t}^{l}(\cdot)}, \overline{B_{t}^{u}(\cdot)}\right.$, $\left.\overline{B_{t}^{e}}\right)$ for $t \in[0, T]$. Extend $\overline{C^{m}}$ naturally to the whole space; then in the exercise region $\overline{\mathcal{E}}$ off the diagonal, $£ \overline{C^{m}}<0$ by the boundary condition. By Lemma 10 , we know that $\overline{C_{t}^{m}}(x, y) \leq C_{t}^{m}(x, y)$; therefore $\overline{C_{t}^{m}}\left(x, B_{t}^{l}(x)\right) \leq C^{m}\left(x, B_{t}^{l}(x)\right)=$ $g\left(x, B_{t}^{l}(x)\right)$. Then $\overline{C_{t}^{m}}\left(x, B_{t}^{l}(x)\right)=g\left(x, B_{t}^{l}(x)\right)$ by the boundary condition. By using the boundary condition of $\left(\bar{C}_{t}^{m}, \bar{B}_{t}^{l}, \bar{B}_{t}^{u}\right)$ again, we have $B_{t}^{l}(x) \geq \bar{B}_{t}^{l}(x)$. Similarly we prove that $B_{t}^{u}(y) \geq \bar{B}_{t}^{u}(y)$. Furthermore, by the definition of $B_{t}^{e}$ and $\bar{B}_{t}^{e}$, we see that $B_{t}^{e} \geq \bar{B}_{t}^{e}$ for the same reason. Write

$$
\begin{aligned}
& A_{v}=\left\{B_{v}^{l}\left(S_{v}^{1}\right) \leq S_{v}^{2}<S_{v}^{1}\right\}, \\
& B_{v}=\left\{B_{v}^{u}\left(S_{v}^{2}\right) \leq S_{v}^{1}<S_{v}^{2}\right\}, \\
& C_{v}=\left\{S_{v}^{1}=S_{v}^{2} \geq B_{v}^{e}\right\}
\end{aligned}
$$


and

$$
\begin{aligned}
& \bar{A}_{v}=\left\{\bar{B}_{v}^{l}\left(S_{v}^{1}\right) \leq S_{v}^{2}<S_{v}^{1}\right\}, \\
& \bar{B}_{v}=\left\{\bar{B}_{v}^{u}\left(S_{v}^{2}\right) \leq S_{v}^{1}<S_{v}^{2}\right\}, \\
& \bar{C}_{v}=\left\{S_{v}^{1}=S_{v}^{2} \geq \bar{B}_{v}^{e}\right\} .
\end{aligned}
$$

Then $A_{v} \subseteq \bar{A}_{v}, B_{v} \subseteq \bar{B}_{v}, C_{v} \subseteq \bar{C}_{v}$. The following claim holds.

Claim 1. The triplet $\left(\overline{C_{t}^{m}}, \overline{B_{t}^{l}(\cdot)}, \overline{B_{t}^{u}(\cdot)}, \overline{B_{t}^{e}}\right)$ satisfies

$$
\begin{aligned}
\overline{C_{t}^{m}}(x, y)= & C_{t}^{e}(x, y) \\
+ & E_{t}^{*}\left[\int_{t}^{T} e^{-r(u-t)}\left\{\left(\delta_{2} S_{u}^{2}-r K\right) \chi_{\left\{\bar{A}_{u}\right\}}+\left(\delta_{1} S_{u}^{1}-r K\right) \chi_{\left\{\bar{B}_{u}\right\}}\right\} d u\right] \\
+ & \frac{1}{2}\left(\sigma_{1}^{2}-2 \sigma_{1} \rho \sigma_{2}+\sigma_{2}^{2}\right) \\
& \times E_{t}^{*}\left[\int_{t}^{T} e^{-r(u-t)} \chi_{\left\{\bar{C}_{u}\right\}} S_{u}^{1} d L\left(u, 0 ; \ln \left(S^{1}\right)-\ln \left(S^{2}\right)\right)\right],
\end{aligned}
$$

where $C_{t}^{e}(x, y)$ denotes the European option value function and $S_{t}^{1}=x, S_{t}^{2}=y$.

Suppose that the claim above has been shown. By Proposition 2(v) and the fact that $B_{t}^{l}(\cdot), B_{t}^{u}(\cdot), B_{t}^{e}$ are nonincreasing functions of time, we have $\delta_{2} S_{u}^{2}-r K \geq 0$ over $A_{u}, \bar{A}_{u}$ and $\delta_{1} S_{u}^{1}-r K \geq 0$ over $B_{u}, \bar{B}_{u}$. The claim and the above relations of $A_{u}, B_{u}$ with $\bar{A}_{u}, \bar{B}_{u}$ imply that $C_{t}^{m}(x, y) \leq \overline{C_{t}^{m}}(x, y)$. So $C_{t}^{m}(x, y)=\overline{C_{t}^{m}}(x, y)$ since we already know $\bar{C}^{m} \leq C^{m}$. Using $\bar{B}_{t}^{l}(x) \leq B_{t}^{l}(x)$ again, and $£ C^{m}=0$ in the continuation regions $\mathcal{C}, \overline{\mathcal{C}}$ and $£ C^{m}<0$ in the exercise regions $\mathcal{E}, \overline{\mathcal{E}}$ (since $\left.C^{m}=\overline{C^{m}}\right)$, we see that $\bar{B}_{t}^{l}(x)=B_{t}^{l}(x)$. Similarly, $\bar{B}_{t}^{u}(\cdot)=B_{t}^{u}(\cdot)$.

Finally we prove Claim 1. Define $Z\left(S_{t}^{1}, S_{t}^{2}, t\right)=e^{-r t} \overline{C_{t}^{m}}\left(S_{t}^{1}, S_{t}^{2}\right)$. Then along the lines of Jacka [(1991), Theorem 4.1], we see that $Z\left(S_{T}^{1}, S_{T}^{2}, T\right)-$ $Z\left(S_{t}^{1}, S_{t}^{2}, t\right)-\int_{t}^{T} e^{-r u} £ \overline{C^{m}} \chi_{\left\{\tau_{u}=u\right\}} d u$ is a martingale. Taking the expectation $E_{t}^{*}$, gives the result stated (i.e., the EEP of $\overline{C^{m}}$ ). This completes the proof of Lemma 11.

Lemma 11 establishes uniqueness under a set of conditions. To prove the uniqueness of the boundary in the context of Theorem 4 we need additional assumptions. To state these, define, for a given set of curves $\beta=\left(\beta_{t}^{l}, \beta_{t}^{u}, \beta_{t}^{e}\right)$, the functions

$$
\begin{aligned}
& \Psi_{\beta}(x, y, t)=C_{t}^{e}(x, y)+\Pi_{t}\left(x, y ; \beta_{t}^{l}, \beta_{t}^{u}, \beta_{t}^{e}\right), \\
& \Phi_{\beta}(x, y, t)=E_{t}^{*}\left[e^{-r\left(\tau_{\beta}-t\right)} g\left(S_{\tau_{\beta}}^{1}, S_{\tau_{\beta}}^{2}\right) \mid S^{1}=x, S^{2}=y\right],
\end{aligned}
$$


where $\tau_{\beta}$ is the first hitting time of the region $\mathcal{C}(\beta)$. We say that $\beta$ satisfies Condition A or B if:

Condition A. $\quad \Psi_{\beta} \geq g(x, y)$ and Itô's lemma can be applied to $\Psi_{\beta}$.

Condition B. $\Phi_{\beta} \geq g(x, y)$ and Itô's lemma can be applied to $\Phi_{\beta}$.

We now prove uniqueness of the exercise boundary in Theorem 4 as follows.

Suppose that Condition A holds. Then, as in the proof of Proposition 9, $\frac{\partial \Psi_{\beta}}{\partial x}, \frac{\partial \Psi_{\beta}}{\partial y}$ are continuous off the diagonal. By using Itô's formula and the BlackScholes principle that $e^{-r t} C_{t}^{e}\left(S_{t}^{1}, S_{t}^{2}\right)$ (i.e., $\left.e^{-r t} \Psi_{\beta}\right)$ is a martingale, we see that the process $U_{t}$ defined by

$$
\begin{aligned}
U_{t} \equiv \int_{0}^{t} e^{-r u}\left(£ \Psi_{\beta} \chi \Subset(\beta)\right. & +\left(£ \Psi_{\beta}+\delta_{2} S_{u}^{2}-r K\right) \chi \varepsilon(\beta, 1) \\
& \left.+\left(£ \Psi_{\beta}+\delta_{1} S_{u}^{1}-r K\right) \chi_{\varepsilon(\beta, 2)}\right) d u \\
& +\int_{0}^{t} e^{-r u}\left(£ \Psi_{\beta}+\frac{\sigma_{1}^{2}-2 \sigma_{1} \rho \sigma_{2}+\sigma_{2}^{2}}{2} S_{u}^{1}\right) \chi_{\varepsilon(\beta, 3)} d L\left(u, 0 ; \ln \left(S^{1}\right)-\ln \left(S^{2}\right)\right)
\end{aligned}
$$

is a local martingale, where $\mathcal{C}(\beta)$ denotes the continuation region determined by $\beta$, and $\mathcal{E}(\beta, i), i=1,2,3$, are the exercise regions determined by $\beta^{l}, \beta^{u}$ and $\beta^{e}$, respectively (e.g., $\left.\mathscr{E}(\beta, 3)=\left\{\left(t, S^{1}, S^{2}\right): S_{u}^{1}=S_{u}^{2} \geq B_{u}^{e}\right\}\right)$. Since the process $U$ is continuous and locally of bounded variation, it must be identically equal to 0 . Note that $\sigma_{1}^{2}-2 \sigma_{1} \rho \sigma_{2}+\sigma_{2}^{2} \geq 0$ and $\beta_{t} \geq r K / \delta_{i}$. Then by the proof in Durrett [(1984), page 221, equation (4)] we see that $£ \Psi_{\beta} \chi \mathcal{C}(\beta)=0$ (otherwise there is a positive probability that $U \neq 0)$. This implies that $\Psi_{\beta}>g(x, y)$ in the continuation region since by assumption $\Psi_{\beta} \geq g(x, y)$.

If Condition B holds, then as in the last step of Lemma 11, we can first prove the EEP representation for $\Phi_{\beta}$, then that $\Phi_{\beta}=\Psi_{\beta}$, and reduce this case to the previous one. Defining $\beta^{e}(t)=\inf \left\{u: \Psi_{\beta}(u, u, t)=g(x, y)\right\}$ enables us to conclude that $\left(\Psi_{\beta}, \beta_{t}^{l}(\cdot), \beta_{t}^{u}(\cdot), \beta^{e}(\cdot)\right)$ is a solution of (12). Hence Theorem 4 follows from Lemma 11.

Proof of Proposition 5. The definition of $C_{t}^{2}\left(S^{1}, S^{2} ; L^{2}\right)$ implies the bound $C_{t}^{2}\left(S^{1}, S^{2} ; L^{2}\right) \leq C_{t}^{m}\left(S^{1}, S^{2}\right)$. It follows that the constrained optimization problem

$$
\max _{L}\left\{C_{t}^{2}\left(S^{1}, S^{2} ; L\right): L \geq S^{2}\right\}
$$

has the solution $L=S^{2}$ when $\left(S^{1}, S^{2}\right) \in \mathcal{E}_{t}^{1}$ (since immediate exercise is optimal). Thus

$$
\left.D^{2}\left(S^{1}, S^{2}\right) \equiv \frac{\partial C_{t}^{2}\left(S^{1}, S^{2}, L\right)}{\partial L}\right|_{L=S^{2}} \leq 0
$$


for all $\left(S^{1}, S^{2}\right) \in \mathcal{E}_{t}^{1}$. We conclude immediately that $L_{t}^{l}\left(S^{1}\right) \equiv \inf \left\{S^{2}: D^{2}\left(S^{1}\right.\right.$, $\left.\left.S^{2}\right) \leq 0\right\} \in \mathcal{C}_{t} \cup B_{t}^{l}\left(S^{1}\right)$, that is, $L_{t}^{l}\left(S^{1}\right) \leq B_{t}^{l}\left(S^{1}\right)$.

Similarly, we can prove that $L_{t}^{u}\left(S_{t}^{2}\right) \leq B_{t}^{u}\left(S_{t}^{2}\right)$.

Acknowledgment. We thank a referee and an Associate Editor for very useful comments.

\section{REFERENCES}

Broadie, M. and Detemple, J. B. (1995). American capped call options on dividend-paying assets. Rev. Financial Stud. 8 161-191.

Broadie, M. and Detemple, J. B. (1996). American option valuation: New bounds, approximations, and a comparison of existing methods. Rev. Financial Stud. 9 1211-1250.

Broadie, M. and Detemple, J. B. (1997). The valuation of American options on multiple assets. Math. Finance 7 241-285.

Broadie, M. and Detemple, J. B. (1999). American options on dividend-paying assets. In Topology and Markets (G. Chichilnisky, ed.) 69-97. Amer. Math. Soc., Providence, RI.

CARr, P., JARRow, R. and MYNENI, R. (1992). Alternative characterizations of American put option. Math. Finance 2 87-106.

DURrett, R. (1984). Brownian Motion and Martingales in Analysis. Wadsworth, Belmont, CA.

El Karoui, N. (1981). Les aspects probabilistes du contrôle stochastique. École d'Été de Probabilités de Saint-Flour IX. Lecture Notes in Math. 876 73-238. Springer, Berlin.

FAKeEv, A. G. (1971). Optimal stopping of a Markov process. Theory Probab. Appl. 16 694-696.

Friedman, A. (1964). Partial Differential Equations of Parabolic Type. Prentice-Hall, Englewood Cliffs, NJ.

Geltner, D., Riddiough, T. and Stojanovic, S. (1996). Insights on the effect of land use choice: The perpetual option on the best of two underlying assets. Journal of Urban Economics 39 20-50.

HARrison, M. and Pliska, S. (1981). Martingales and stochastic integrals in the theory of continuous trading. Stochastic Process. Appl. 11 215-260.

JACKA, S. D. (1991). Optimal stopping and the American put. Math. Finance 1 1-14.

JACOD, J. (1979). Calcul stochastique et problèmes de martingales. Lecture Notes in Math. 714. Springer, Berlin.

JAILlET, P., LAMBERTON, D. and LAPEYRE, B. (1990). Variational inequalities and the pricing of American option. Acta Appl. Math. $21263-289$.

Jamshidian, F. (1992). An analysis of American options. Review of Futures Markets 11 72-80.

Johnson, H. (1987). The options on the maximum of the minimum of several assets. Journal of Financial Quantitative Analysis 22 227-283.

Karatzas, I. (1988). On the pricing of American options. Appl. Math. Optim. 17 37-60.

Karatzas, I. and Shreve, S. (1987). Brownian Motion and Stochastic Calculus. Springer, New York.

KIM, I. J. (1990). The analytic valuation of American options. Rev. Financial Stud. 3 547-572.

Krylov, N. V. (1980). Controlled Diffusion Process. Springer, New York.

MCKeAN, H. P. (1965). A free boundary problem for the heat equation arising from a problem in mathematical economics. Industrial Management Review 6 32-39.

RUTKowski, M. (1994). The early exercise premium representation of foreign market American options. Math. Finance 4 313-325.

TAN, K. and VetZaL, K. (1995). Early exercise regions for exotic options. Journal of Derivatives $342-56$. 
VAn Moerbeke, P. L. (1976). On optimal stopping and free boundary problems. Arch. Rational Mech. Anal. 60 101-148.

VilleneuVE, S. (1999). Exercise region of American rainbow options. Finance and Stochastics 3 295-322.

\section{J. Detemple}

SCHOOL OF MANAGEMEnt 546E

BOSTON UNIVERSITY

595 Commonwealth Ave.

Boston, Massachusetts 02215

E-MAIL: detemple@bu.edu
S. FENG

DEPARTMENT OF MATHEMATICS

AND STATISTICS

MCMASTER UNIVERSITY

HAMILTON, ONTARIO

L8S 4K1 CANADA

E-MAIL: shuifeng@mcmail.mcmaster.ca

W. TIAN

CEntre For Advanced Studies in Finance

UNIVERSITY OF WATERLOO

WATERLOO, ONTARIO

N2L 3G1 CANADA

AND

TD SECURITIES

79 WELLINGSTON STREET

TORONTO, ONTARIO

M5K 1A1 CANADA

E-MAIL: weidong.tian@tdsecurities.com 